\title{
HMGB1 Acts on Microglia Mac1 to Mediate Chronic Neuroinflammation That Drives Progressive Neurodegeneration
}

\author{
Hui-Ming Gao, Hui Zhou, Feng Zhang, Belinda C. Wilson, Wayneho Kam, and Jau-Shyong Hong \\ Laboratory of Toxicology and Pharmacology, National Institute of Environmental Health Sciences/National Institutes of Health, Research Triangle Park, \\ North Carolina 27709
}

What drives the gradual degeneration of dopamine neurons in Parkinson's disease (PD), the second most common neurodegenerative disease, remains elusive. Here, we demonstrated, for the first time, that persistent neuroinflammation was indispensible for such a neurodegenerative process. 1-Methyl-4-phenylpyridinium, lipopolysaccharide (LPS), and rotenone, three toxins often used to create PD models, produced acute but nonprogressive neurotoxicity in neuron-enriched cultures. In the presence of microglia (brain immune cells), these toxins induced progressive dopaminergic neurodegeneration. More importantly, such neurodegeneration was prevented by removing activated microglia. Collectively, chronic neuroinflammation may be a driving force of progressive dopaminergic neurodegeneration. Conversely, ongoing neurodegeneration sustained microglial activation. Microglial activation persisted only in the presence of neuronal damage in LPS-treated neuron- glia cultures but not in LPS-treated mixed-glia cultures. Thus, activated microglia and damaged neurons formed a vicious cycle mediating chronic, progressive neurodegeneration. Mechanistic studies indicated that HMGB1 (highmobility group box 1), released from inflamed microglia and/or degenerating neurons, bound to microglial Macl (macrophage antigen complex 1) and activated nuclear factor- $\kappa$ B pathway and NADPH oxidase to stimulate production of multiple inflammatory and neurotoxic factors. The treatment of microglia with HMGB1 led to membrane translocation of $\mathrm{p}^{7} 7^{\text {phox }}$ (a cytosolic subunit of NADPH oxidase) and consequent superoxide release, which required the presence of Mac1. Neutralization of HMGB1 and genetic ablation of Mac1 and $\operatorname{gp} 91^{\text {phox }}$ (the catalytic submit of NADPH oxidase) blocked the progressive neurodegeneration. Our findings indicated that HMGB1Mac1-NADPH oxidase signaling axis bridged chronic neuroinflammation and progressive dopaminergic neurodegeneration, thus identifying a mechanistic basis for chronic PD progression.

\section{Introduction}

Gradual, irreversible loss of dopamine (DA) neurons in the substantia nigra $(\mathrm{SN})$ is the signature lesion of Parkinson's disease (PD). When $50-60 \%$ of nigral DA neurons are lost, clinical symptoms of PD become apparent. PD progresses insidiously for 5-7 years (preclinical period) and then continues to worsen even under the symptomatic treatment. The formation of $\alpha$-synuclein-containing Lewy body, the pathological hallmark of PD, in grafted fetal mesencephalic neurons in PD patients strongly suggests the existence of a driving mechanism by which PD gradually progresses and even propagates from host to transplanted cells (Kordower et al., 2008; Li et al., 2008; Mendez et al., 2008). Unfortunately, little is known about what drives PD to unstoppable progression. As a result, no proven clinical therapeutics is now available to retard PD progression.

\footnotetext{
Received July 16, 2010; revised Nov. 1, 2010; accepted Nov. 6, 2010.

This work was supported by the Intramural Research Program of the National Institutes of Health, the National Institute of Environmental Health Sciences. We thank Anthony Lockhart for assistance with animal colony management and maintenance of the timed pregnant mice.

The authors declare no competing financial interests.

Correspondence should be addressed to Hui-Ming Gao, National Institutes of Health/National Institute of Environmental Health Sciences, 111 T. W. Alexander Drive, Research Triangle Park, NC 27709. E-mail: ga02@ niehs.nih.gov.

DOI:10.1523/JNEUROSCI.3732-10.2011

Copyright $\odot 2011$ the authors $\quad 0270-6474 / 11 / 311081-12 \$ 15.00 / 0$
}

Therefore, to discern mechanism(s) responsible for PD progressivity is urgent and of paramount importance.

Multiple pathological events, including oxidative stress, mitochondrial dysfunction, excitatory toxicity, and protein aggregation, are implicated in the pathogenesis of PD (McNaught and Olanow, 2006; Martinez-Vicente et al., 2008; Zhou et al., 2008). Recently, the inflammatory mechanism in PD and other neurodegenerative diseases is becoming increasingly recognized (McGeer et al., 1988; Kreutzberg, 1996; Gao and Hong, 2008; Monahan et al., 2008; Hirsch and Hunot, 2009; Przedborski, 2010). DNA polymorphisms of several inflammatory cytokines might become risk factors for PD (Wahner et al., 2007). The SN of PD patients and animal models revealed activated microglia and accumulation of inflammatory mediators (McGeer et al., 2003, Block et al., 2007). Moreover, suppression of inflammation correlates with less neuronal impairment in various PD models (Du et al., 2001; Gao et al., 2003b; Zhang et al., 2010). Recently developed in vivo models by our group indicated that chronic neuroinflammation was able to induce delayed and progressive DA degeneration (Gao et al., 2002b; Qin et al., 2007). Taking advantage of multiple in vitro PD models created by one inflammogen and two neurotoxins, we now further investigate whether, unlike other PD pathologic contributors, excessive chronic neu- 
roinflammation is a major driving force of chronic PD neurodegeneration. We also examine cellular and molecular mechanisms by which initial neuronal damages are transformed into chronic progressive neurodegeneration and chronic neuroinflammation is maintained in PD.

High-mobility group box 1 (HMGB1), a non-histone DNAbinding protein, modulates interactions of various transcription factors with DNA in nuclei. It is of special interest that HMGB1 can be actively secreted from inflammatory cells or passively released from necrotic cells to initiate inflammatory responses (Lotze and Tracey, 2005). Although extracellular cytokine-like effects of HMGB1 have been increasingly appreciated in recent years, the very limited publications regarding its effects on neuron survival/death were mostly conducted in acute or subacute experimental settings. In the present study, we pinpointed HMGB1 as a main mediator bridging persistent neuroinflammation and chronic, progressive neurodegeneration.

\section{Materials and Methods}

Primary neuronal and glial cultures. Mesencephalic neuron-glia or neuronenriched cultures were prepared from the ventral mesencephalon of embryonic day $14 \pm 0.5$ Fischer 334 rats, B6.129S4-Itgam ${ }^{\text {tm1Myd } / J ~}\left(\mathrm{Macl}^{-/-}\right)$ mice, B6.129S6-Cybb ${ }^{\text {tm1Din }} / \mathrm{J}$ ( $g p 91^{\text {PHOX-/- }}$, NADPH oxidase-deficient) mice, or wild-type (C57BL/6J) mice. Both neuron-glia and neuronenriched cultures were maintained in MEM supplemented with $10 \%$ heatinactivated fetal bovine serum (FBS) and 10\% heat-inactivated horse serum (HS), $1 \mathrm{~g} / \mathrm{L}$ glucose, $2 \mathrm{~mm}$ L-glutamine, $1 \mathrm{~mm}$ sodium pyruvate, and $0.1 \mathrm{~mm}$ nonessential amino acids. For neuron-enriched cultures, $2 \mathrm{~d}$ after initial seeding, cytosine $\beta$-D-arabinofuranoside $(8 \mu \mathrm{M})$ was added into the cultures to suppress the proliferation of glia. Seven-day-old cultures were treated with vehicle or desirable reagents in treatment medium (MEM containing $2 \%$ FBS, $2 \% \mathrm{HS}, 2 \mathrm{~mm}$ L-glutamine, and $1 \mathrm{~mm}$ sodium pyruvate). At the time of treatment, the neuron-glia cultures were made up of 12\% microglia, $49 \%$ astrocytes, and $39 \%$ neurons of which $2.5-3.5 \%$ were tyrosine hydroxylase (TH)-immunoreactive (IR) neurons; the neuron-enriched cultures consisted of $4 \%$ astrocytes, $<0.1 \%$ microglia, and $96 \%$ neurons of which $3.0-3.8 \%$ were TH-IR neurons. In either neuronenriched cultures or neuron-glia cultures, the cell composition was not different among different genotypes (Gao et al., 2002a).

Mixed-glia cultures were prepared from whole brains of postnatal day 1 rats. Disassociated brain cells were seeded onto 24 -well $\left(4 \times 10^{5} /\right.$ well $)$ culture plates and maintained in $1 \mathrm{ml} /$ well DMEM/F-12 supplemented with $10 \%$ FBS, $2 \mathrm{~mm}$ L-glutamine, $1 \mathrm{~mm}$ sodium pyruvate, and $0.1 \mathrm{~mm}$ nonessential amino acids. The medium was changed every $3 \mathrm{~d}$. When reaching confluence at 11-12 d after plating, the cultures contained $\sim 80 \%$ glial fibrillary acidic protein-IR astrocytes and $\sim 20 \%$ ionized calcium binding adapter molecule 1 (Iba1)-IR microglia and were used for treatment. Microglia-enriched cultures were prepared from whole brains of 1-d-old rodents following a previous protocol. Microglia were isolated by shaking the flasks containing confluent mixed-glia cultures for $3 \mathrm{~h}$ at $150 \mathrm{rpm}$ (Gao et al., 2002b).

Reconstituted cell cultures. Two different types of reconstituted cultures containing microglia and neurons were prepared: enriched microglia were either directly plated on top of the existing neuron-enriched cultures (Gao et al., 2003a) or plated into transwells placed above the neuron layer. Briefly, midbrain neuron-enriched cultures were first prepared in regular 24-well culture plates. Six days after the initial seeding, the cultures were changed to fresh neuron-glia treatment medium. Transwells (HTS 24-well multiwell insert systems, $1.0 \mu \mathrm{m}$ pore size, polyethylene terephthalate membrane) were inserted into the culture plates. Then highly enriched microglia $\left(5 \times 10^{4} /\right.$ well $)$ were added to the transwells. One day later, the reconstituted cultures were treated with lipopolysaccharide (LPS) or vehicle. The transwells were removed $24 \mathrm{~h}$ after the treatment, and the culture media were either kept or changed to fresh treatment medium. The removed transwells with treated microglia were then put into parallel plates containing neuron-enriched cultures. In another treatment group, the transwells were kept in the cultures until
A

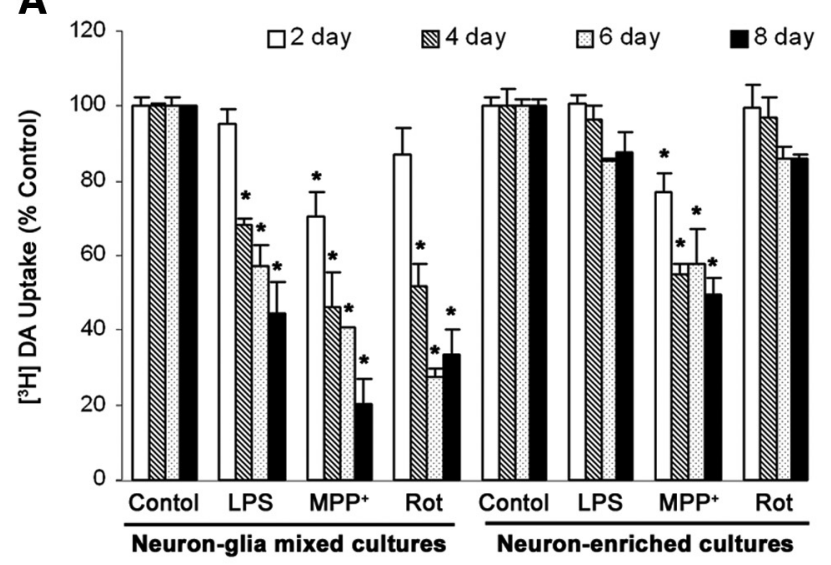

B

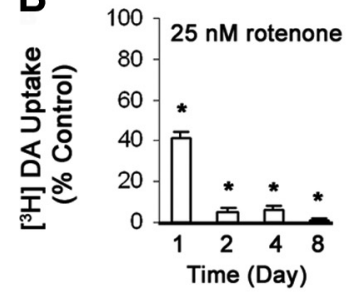

D

C

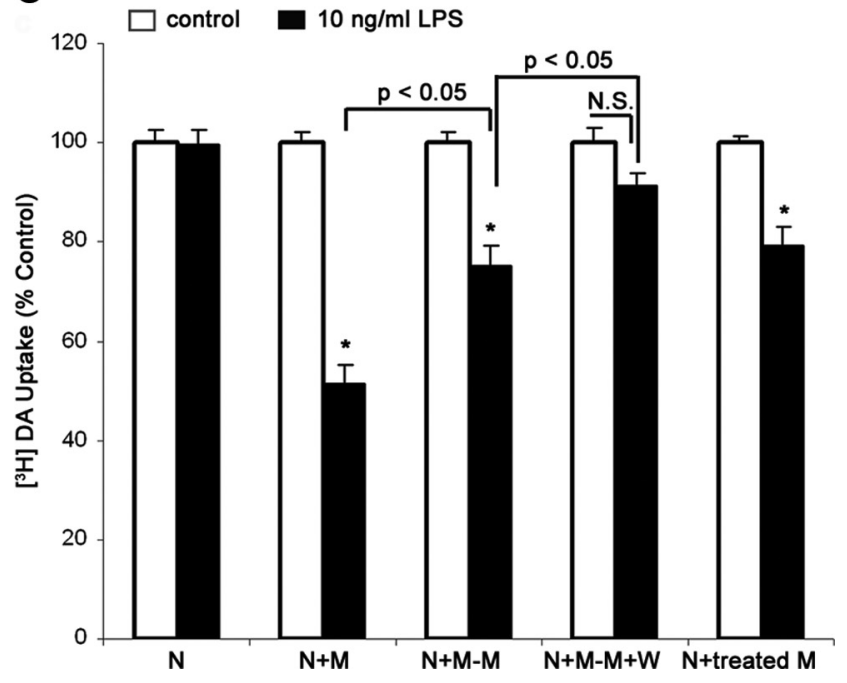

Figure 1. Progressive DA neurodegeneration depended on the presence of microglia. $A$, Time course dependence for the effect of LPS, MPP ${ }^{+}$, and rotenone on DA uptake capacity in the mesencephalic neuron-glia cultures but not in the neuron-enriched cultures. Cultures were treated with vehicle, $10 \mathrm{ng} / \mathrm{ml} \mathrm{LPS}, 0.5 \mu \mathrm{m} \mathrm{MPP}{ }^{+}$, or $10 \mathrm{~nm}$ rotenone for $2-8 \mathrm{~d}$ and then assayed for $\left[{ }^{3} \mathrm{H}\right] \mathrm{DA}$ uptake. After 2, 4, 6, and $8 \mathrm{~d}$ posttreatment, the amount of DA uptake in vehicle-treated neuron-glia cultures was $0.79 \pm 0.14,0.81 \pm 0.04,0.690 \pm 0.10$, and $0.60 \pm 0.06 \mathrm{pmol} / \mathrm{min}$ per well, respectively; in vehicle-treated neuron-enriched cultures, it was $0.88 \pm 0.06,0.86 \pm 0.15,0.82 \pm 0.10$, and $0.79 \pm 0.11 \mathrm{pmol} / \mathrm{min}$ per well, respectively. $\boldsymbol{B}$, Treatment of neuron-enriched cultures with a high dose of rotenone $(25 \mathrm{~nm})$ caused a quick and dramatic, but nonprogressive, reduction in DA uptake. $\boldsymbol{C}, \boldsymbol{D}$, Removal of activated microglia rescued DA neurons from progressive degeneration. The neuron-enriched cultures $(N)$ and reconstituted cultures $(N+M)$ containing microglia $\left(5 \times 10^{4}\right.$ microglia/well) in transwells and enriched neuron layer underneath were treated with vehicle or 10 $\mathrm{ng} / \mathrm{ml}$ LPS for $24 \mathrm{~h}$. The transwells with treated microglia were then removed from the original culture plates $(\mathrm{N}+\mathrm{M}-\mathrm{M})$ and put into sister culture plates with existing neuron-enriched cultures $(\mathrm{N}+$ treatedM). The survival of DA neurons was assessed by [ $\left.{ }^{3} \mathrm{H}\right] \mathrm{DA}$ uptake immediately (D) or6 d later ( $(\boldsymbol{C}$. The results are expressed as a percentage of the time-matched control cultures and are the mean \pm SEM of three to four experiments performed in triplicate. ${ }^{*} p<0.05$ compared with the time-matched vehicle-treated controls $(\boldsymbol{A}, \boldsymbol{B})$ or the corresponding vehicle-treated controls $(\boldsymbol{C}, \boldsymbol{D}) . p<0.05$ was considered statistically significant. Rot, Rotenone; $\mathrm{M}$, microglia; $\mathrm{N}+\mathrm{M}-\mathrm{M}+\mathrm{W}$, wash the neuron layer after the removal of transwells; N.S., not significant. 
A

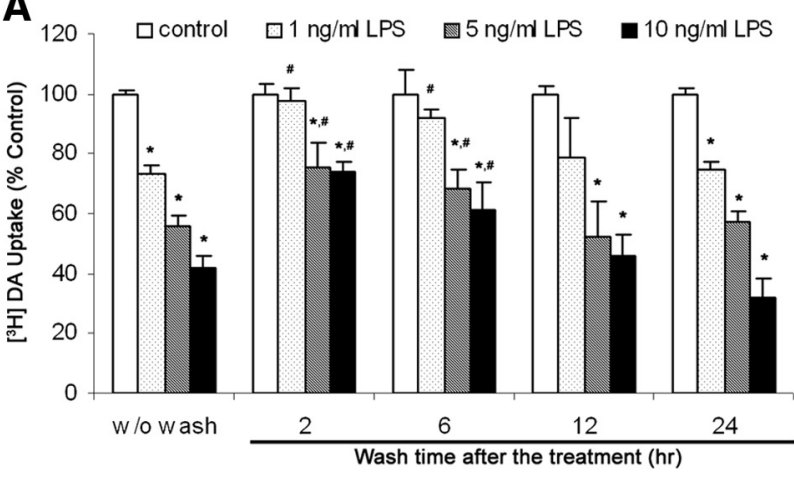

C

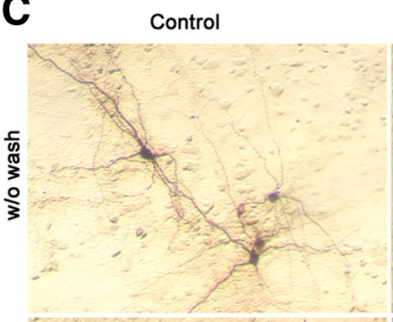

$10 \mathrm{ng} / \mathrm{ml}$ LPS
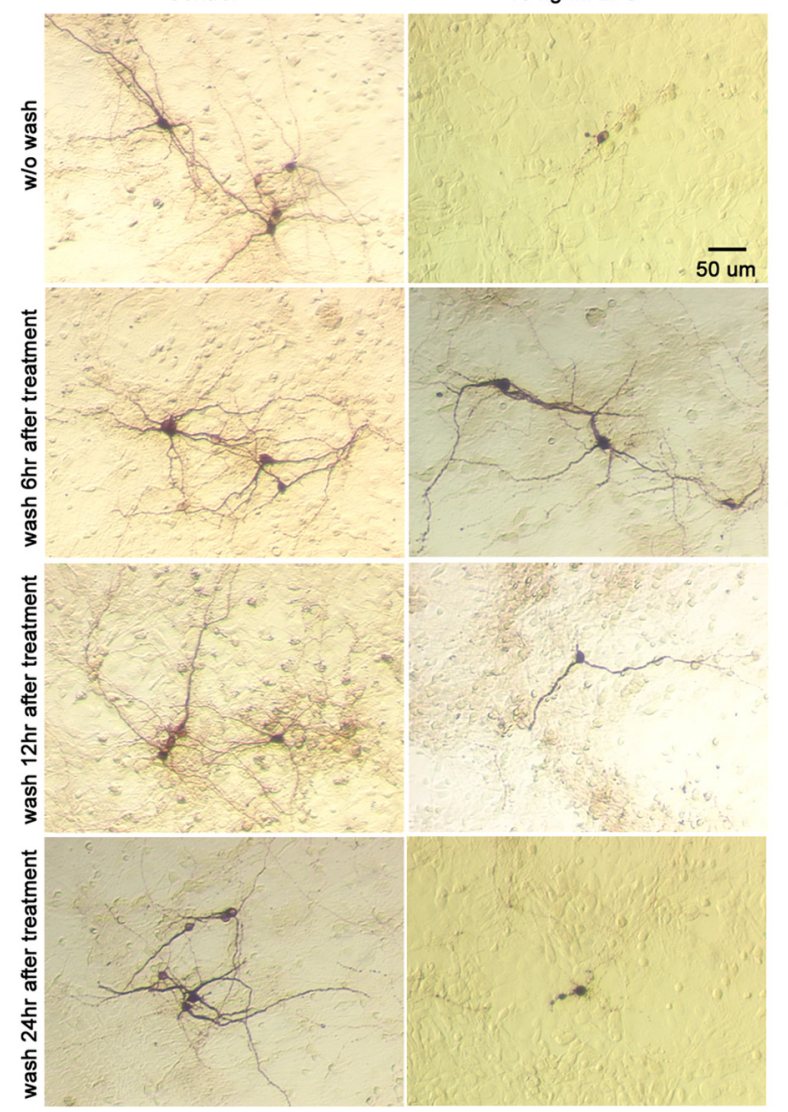

$E$

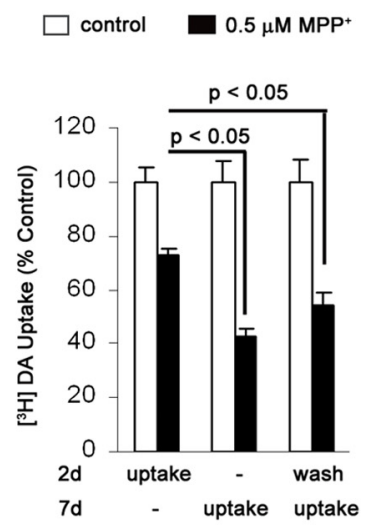

Figure 2. Effects of washout of LPS/MPP ${ }^{+}$and released inflammatory/neurotoxic factors on DA neurodegeneration. $A, D, A t$ specified time points, the culture media were completely aspirated and the neuron- glia cultures were washed twice and replenished with fresh treatment media. DA neuron survival was determined with [ $\left.{ }^{3} \mathrm{H}\right] \mathrm{DA}$ uptake assay $(\boldsymbol{A})$ or quantification of TH-IR neurons $(\boldsymbol{D}) 7 \mathrm{~d}$ after the initial treatment. Compared with time-matched controls, LPS-treated cultures showed significant neuronal damage even after the wash. $\boldsymbol{B}$, There was no significant reduction in [ $\left.{ }^{3} \mathrm{H}\right] \mathrm{DA}$ uptake within $48 \mathrm{~h}$ after LPS treatment of neuron-glia cultures with $10 \mathrm{ng} / \mathrm{ml}$ LPS. C, Representative images from three experiments confirmed the neuronal loss and dendrite degeneration of DA neurons even after drug washout and inflammatory factor withdrawal at different time points. $\boldsymbol{E}$, Neuron-glia cultures were treated with vehicle or $0.5 \mu \mathrm{MMPP}{ }^{+}$. Two days after the treatment, the cells were washed twice and refed with fresh treatment media. DA uptake assay was performed 2 or $7 \mathrm{~d}$ after the initial treatment. Notably, the drug washout at $2 \mathrm{~d}$ after $\mathrm{MPP}^{+}$treatment could not stop DA neurons from additional degeneration. Results are expressed as a percentage of the time-matched controls and are the mean \pm SEM of four experiments performed in triplicate. ${ }^{*} p<0.05$ compared with the time-matched vehicle-treated controls. ${ }^{\#} p<0.05$ compared with the LPS-treated cultures without wash. $p<0.05$ was considered statistically significant. N.S., Not significant.

uptake assay. Seven days after LPS/vehicle treatment, the degeneration of DA neurons was assessed by $\left[{ }^{3} \mathrm{H}\right] \mathrm{DA}$ uptake.

High-affinity $\left[{ }^{3} \mathrm{H}\right]$ dopamine uptake assay. Uptake assays were determined by incubation of cultures for $15 \mathrm{~min}$ at $37^{\circ} \mathrm{C}$ with $1 \mu \mathrm{M}\left[{ }^{3} \mathrm{H}\right] \mathrm{DA}$ (30 Ci/mmol; NEN) as described previously (Gao et al., 2002b). Nonspecific uptake was determined in the presence of $10 \mu \mathrm{M}$ mazindol. $\left[{ }^{3} H\right]$ LPS binding and internalization assay. Rat mesencephalic neuron-glia cultures were incubated with treatment media containing $0.01 \mu \mathrm{Ci}\left[{ }^{3} \mathrm{H}\right]$ LPS $(10 \mathrm{ng} / \mathrm{ml})$ for $24 \mathrm{~h}$ at 37 or $4^{\circ} \mathrm{C}$. Afterward, the medium was removed and cells were washed twice with fresh treatment media. Cells were then lysed with $250 \mu$ of $1 \mathrm{~N}$ $\mathrm{NaOH}$. The removed culture media, the first and second washing media, and cell lysate were counted for radioactivity.

Measurement of superoxide, nitric oxide, and cytokines. The production of superoxide was determined by measuring the superoxide dismutase (SOD)-inhibitable reduction of tetrazolium salt, WST-1, as described previously with modifications. Seven-day-old mesencephalic neuron-glia cultures grown in 96-well plates were treated with LPS or vehicle in 150 $\mu l$ of phenol red-free treatment medium. At 30 min or $0.5-5 \mathrm{~d}$ after the treatment, $50 \mu \mathrm{l}$ of WST-1 (1 mM) in treatment medium with and without $800 \mathrm{U} / \mathrm{ml}$ SOD was added. The cultures were incubated for $20 \mathrm{~min}$ at $37^{\circ} \mathrm{C}$. The absorbance at $450 \mathrm{~nm}$ was read with a SpectraMax Plus microplate spectrophotometer (Molecular Devices). The production of nitric oxide (NO) was determined using Griess reagent. The levels of tumor necrosis factor- $\alpha$ $(\mathrm{TNF}-\alpha)$ and interleukin-1 $\beta$ (IL-1 $\beta$ ) in the culture medium were measured with commercial ELISA kits from R \& D Systems.

Immunostaining and cell counting. Immunostaining was performed as described previously (Gao et al., 2002b) with antibodies specific for TH (a gift from Dr. John Reinhard, GlaxoSmithKline, Research Triangle Park, NC) or F4/80 (Serotec). Images were recorded with a CCD camera and the MetaMorph software (Molecular Devices). For visual enumeration of the immunostained cells, images from 10 representative areas per well were taken, and the TH-IR neurons were counted. For each experiment, three to four wells per treatment condition were used, and results from three to four independent experiments were obtained.

Gel electrophoresis and Western blotting analysis. The protein extracts from cultured cells were homogenized in radioimmunoprecipitation assay (RIPA) lysis buffer $(50 \mathrm{~mm}$ Tris-HCl, pH 8.0, 150 mm NaCl, 5 mm EDTA, $1 \%$ NP-40, 0.5\% sodium deoxycholate, $0.1 \%$ SDS, and 1:100 protease inhibitor cocktail), sonicated, and heated to $100^{\circ} \mathrm{C}$ for $10 \mathrm{~min}$. Protein concentrations were determined using the biocinchoninic acid assay (Pierce). Protein samples were resolved on $4-12 \%$ SDS-PAGE gels, and immunoblot analyses were performed using antibodies against Iba1 (1:2500; Wako Pure Chemicals), inducible nitric oxide synthase (iNOS) (1:1000; BD Biosciences), HMGB1 (1:2000; Abcam), macrophage antigen complex 1 (Mac1) (1:1000; Abcam), or gp91 ${ }^{\text {phox }}$ (1:2000; BD Biosciences). An antibody against $\beta$-actin (1:5000; Cell Signaling Technology) was included as an internal standard to monitor loading errors. Immunoprecipitation. Primary microglia $\left(2-5 \times 10^{6}\right)$ were treated with $500 \mathrm{ng} / \mathrm{ml} \mathrm{HMGB1}$ or vehicle at $4^{\circ} \mathrm{C}$ for $2 \mathrm{~h}$ or $37^{\circ} \mathrm{C}$ for $15 \mathrm{~min}$, followed by three washes with ice-cold PBS. Cell lysates were prepared in TNE buffer (10 mm Tris-HCl at pH 7.5, 1\% Nonidet P-40, 0.15 M NaCl, 1 mM 
EDTA, and 1:100 protease inhibitor cocktail) and centrifuged at $17,400 \times g$ for $20 \mathrm{~min}$ at $4^{\circ} \mathrm{C}$. The supernatant was preincubated with protein ASepharose beads (GE Healthcare) for $2 \mathrm{~h}$ at $4^{\circ} \mathrm{C}$ and centrifuged. After this preclear process, the supernatant was incubated at $4^{\circ} \mathrm{C}$ with antiHMGB1 antibody ( $2 \mu \mathrm{g}$; Abcam) or anti-Mac1 antibody (2.5 $\mu \mathrm{g}$; Abcam) overnight and with protein A-Sepharose slurry for $2 \mathrm{~h}$. Protein A beads were collected by centrifugation at $2000 \times$ $g$ for $5 \mathrm{~min}$ and were washed six times with TNE buffer. Bound proteins were eluted in sample buffer, separated on 4-12\% SDS-PAGE gels, and blotted with anti-Mac1 and anti-HMGB1 antibodies.

Membrane fractionation. $\mathrm{Macl}^{-1-}$ and wildtype mouse microglia were lysed in hypotonic lysis buffer (1 mm EGTA, 1 mм EDTA, $10 \mathrm{~mm}$ $\mathrm{NaF}, 2 \mathrm{~mm} \mathrm{MgCl}_{2}, 10 \mathrm{~mm}$ DTT, 1 mm sodium orthovanadate, $10 \mathrm{~mm} \beta$-glycerophosphate, $0.32 \mathrm{~m}$ sucrose, $1 \mathrm{~mm}$ PMSF, and protease inhibitor cocktail) and incubated on ice for 30 min. After Dounce homogenization (tight pestle A, 20-25 stokes), the lysates were centrifuged at $3000 \times g$ for $20 \mathrm{~min}$ at $4^{\circ} \mathrm{C}$ to remove unbroken cells, cell debris, and nuclei. The supernatants were centrifuged at $185,000 \times g$ for $60 \mathrm{~min}$ at $4^{\circ} \mathrm{C}$ to separate the total membrane fraction from all soluble proteins. The pellets were washed once, followed by high-speed sedimentation $(185,000 \times g)$. The pellets solubilized in the TNE buffer were used as membrane fraction.

Nuclear and cytosolic fractionation. Rat microglia were incubated with $500 \mathrm{ng} / \mathrm{ml} \mathrm{HMGB1}$ or vehicle at $37^{\circ} \mathrm{C}$ for 15 or $30 \mathrm{~min}$ and washed with cold PBS. All of the following steps were performed at $4^{\circ} \mathrm{C}$, and protease inhibitor cocktail was included in all buffers (Wang et al., 2003). After centrifugation at $2000 \times g$ for $10 \mathrm{~min}$, cells pellets were suspended in buffer A $(10 \mathrm{~mm}$ HEPES at $\mathrm{pH} 8,1.5 \mathrm{~mm} \mathrm{MgCl}_{2}, 10 \mathrm{~mm} \mathrm{KCl,} 1 \mathrm{~mm}$ dithiothreitol, and $1 \mathrm{~mm}$ PMSF) and incubated on ice for $15 \mathrm{~min}$. Cells were then homogenized with a loose-fitting Dounce homogenizer (pestle $\mathrm{B})$, and the lysis completion was monitored by trypan blue staining. The homogenate was centrifuged at $3000 \times g$ for $15 \mathrm{~min}$. The supernatant was then centrifuged for $60 \mathrm{~min}$ at $100,000 \times g$. The resultant supernatant was used as cytosolic fraction. The recovered nuclei were washed twice with buffer B ( $60 \mathrm{~mm} \mathrm{NaCl}, 10 \mathrm{~mm}$ HEPES at $\mathrm{pH}$ 8.0, 25\% glycerol, $0.1 \mathrm{~mm}$ EDTA, 1 mM dithiothreitol, and $1 \mathrm{~mm}$ PMSF). The washed nuclear pellets were extracted with buffer C (20 mM HEPES, 25\% glycerol, $0.42 \mathrm{M} \mathrm{NaCl}, 1.5 \mathrm{~mm} \mathrm{MgCl}, 0.2 \mathrm{~mm}$ EDTA, 1 mm dithiothreitol, and $1 \mathrm{~mm}$ PMSF). The nuclear suspension was centrifuged at 25,000 $\times g$ for 30 min, and the supernatant was collected as nuclear fraction.

Statistical analysis. All values are expressed as the mean \pm SEM. Differences among means were analyzed using one- or two-way ANOVA with treatment as the independent factors. When ANOVA showed significant differences, pairwise comparisons between means were tested by Newman-Keuls post hoc testing. In all analyses, the null hypothesis was rejected at the 0.05 level.

\section{Results}

Microglia were required for progressive

DA neurodegeneration

A great amount of evidence indicates that overactivation of microglia is an important contributor to the demise of DA neurons in PD.
B

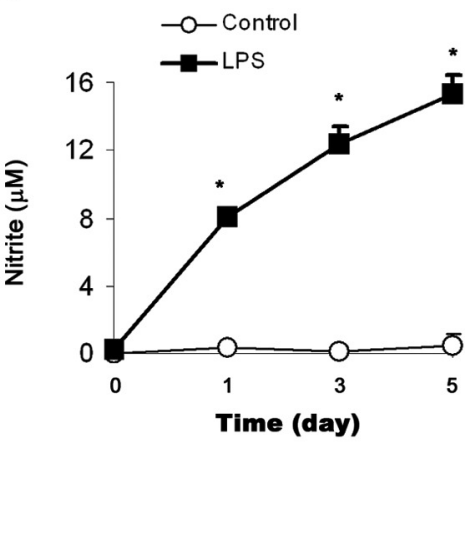

E

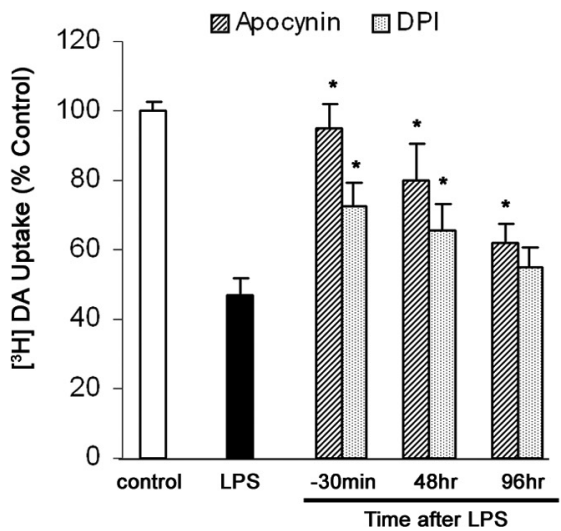

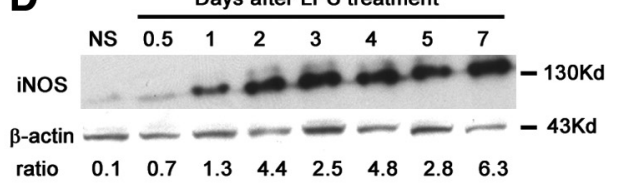

$\mathbf{F}$

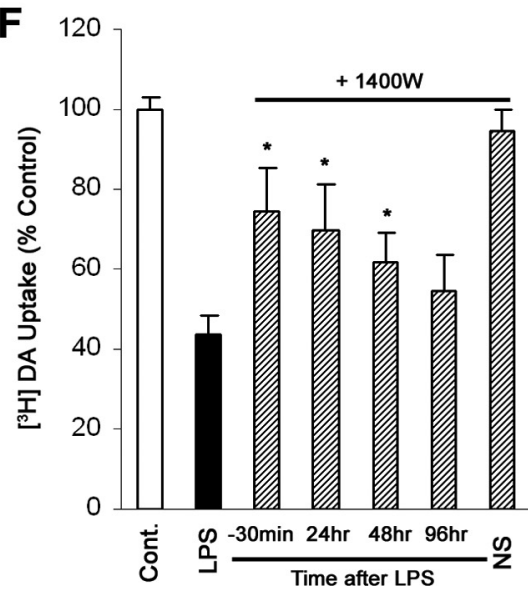

Figure 3. Long-lasting secretion of superoxide and NO from activated microglia in mediating progressive DA neurodegeneration. $\boldsymbol{A}, \boldsymbol{B}$, Continuing release of superoxide and nitrite (an indicator of $\mathrm{N} 0$ production) in midbrain neuron- glia cultures after LPS treatment. The release of superoxide was measured as SOD-inhibitable WST-1 reduction. ${ }^{*} p<0.05$ compared with the timematched control cultures. C, D, Persistent upregulation of gp $91^{\text {phox }}$ and iNOS was observed after the neuron- glia cultures were three experiments. $\boldsymbol{E}, \boldsymbol{F}, \mathrm{NADPH}$ oxidase inhibitors apocynin or DPI and iNOS inhibitor $1400 \mathrm{~W}$ protected DA neurons against -mediated chronic neurodegeneration, when neuron - glia cultures were pretreated for $30 \mathrm{~min}$, or posttreated at $48 \mathrm{~h}$. Here, DA uptake was conducted $7 \mathrm{~d}$ after initial LPS treatment. ${ }^{*} p<0.05$ compared with the corresponding LPS-treated cultures. Results shown above are the mean \pm SEM of three to four experiments performed in triplicate. NS, Normal saline.

To address whether microglial activation is indispensable for the progressive neuronal loss in $\mathrm{PD}$, we first compared the survival of DA neurons in the presence or absence of microglia after the cultures were stimulated with three "PD-producing toxins." Inflammogen LPS was used to initiate direct immunologic insults and consequent neurodegeneration. Neurotoxin 1-methyl4-phenylpyridinium $\left(\mathrm{MPP}^{+}\right)$was used to trigger direct neuronal lesions and to induce a secondary microglial reaction (Gao et al., 2003a). $\mathrm{MPP}^{+}$is a toxic metabolite of 1-methyl-4-phenyl-1,2,3,6tetrahydropyridine (MPTP) that causes parkinsonism in both human and nonhuman primates (Langston et al., 1999; McGeer et al., 2003). Rotenone, a common pesticide, reproduces parkinsonism when chronically administered to rodents (Betarbet et al., 2000); mechanistically, both impairment of neuronal mitochondrial complex I and activation of microglia participate in rotenone-induced destruction of DA neurons (Betarbet et al., 2000; Gao et al., 2002a). 
We found that, in neuron-glia cultures that contain neurons, astroglia, and microglia, all three toxins induced progressive degeneration of DA neurons, as indicated by time-dependent decreases in $\left[{ }^{3} \mathrm{H}\right] \mathrm{DA}$ uptake after the treatment. In contrast, in neuron-enriched cultures, $\mathrm{MPP}^{+}(0.5 \mu \mathrm{M})$ and rotenone $(25 \mathrm{nM})$ caused acute but nonprogressive neurotoxicity (Fig. $1 A, B$ ). Specifically, $\mathrm{MPP}^{+}(0.5 \mu \mathrm{M})$ induced a 25 and $45 \%$ reduction in DA uptake after 2 and $4 \mathrm{~d}$ treatment, respectively; the neurotoxicity failed to progress further after prolonged treatment (Fig. 1 A). Similarly, treatment of neuron-enriched cultures with a high dose of rotenone ( $25 \mathrm{~nm}$ ) caused a quick and dramatic, but nonprogressive, neurotoxicity as shown by a $60 \%$ reduction in DA uptake $1 \mathrm{~d}$ after the treatment (Fig. $1 B$ ). Rotenone at $10 \mathrm{nM}$ concentration did not show neurotoxicity in neuron-enriched cultures, which otherwise was toxic to DA neurons in neuronglia cultures, indicating the presence of microglia enhanced rotenone neurotoxicity. These data together indicate that activated microglia were required for chronic, progressive neurodegeneration, regardless the nature of initial insults (inflammogen or neurotoxin).

\section{Withdrawal of activated microglia prevented DA neurons from progressive degeneration}

To more rigorously evaluate the dependence of progressive DA neurodegeneration on microglial activation, we generated reconstituted cell cultures using transwells and separated activated microglia from neurons after the cultures were treated with LPS for $24 \mathrm{~h}$. Although there was no physical contact between neurons and microglia in these reconstituted cultures, secreted soluble factors could move across the transwell membranes. As shown in Figure $1 C$, the treatment of neuron-enriched cultures with LPS for $7 \mathrm{~d}$ did not reduce DA uptake, which indicates no microglia contamination in neuronal layer of these reconstituted cultures. In the presence of microglia in the transwells, LPS treatment for $7 \mathrm{~d}$ decreased the DA uptake capacity by $50 \%$. Removal of activated microglia $24 \mathrm{~h}$ after LPS treatment significantly attenuated DA neurodegeneration observed $7 \mathrm{~d}$ after LPS addition. Withdrawal of activated microglia and washout of their secreted inflammatory factors completely abrogated LPS neurotoxicity. Moreover, the reduction in DA uptake in neuron-enriched cultures supplemented with activated microglia implies that activated microglia in certain degrees could continue to release some neurotoxic factors leading to neuronal damage. The amount of DA uptake in reconstituted cultures treated for $24 \mathrm{~h}$ with vehicle and LPS (10 ng/ml LPS) was $100 \pm 1.4$ and $98 \pm 3.2 \%$, respectively (Fig. 1D), which suggests no preexisting neuron demise before the removal of activated microglia. These findings further support the essential role of persistent activation of microglia for progressive neurodegeneration.

\section{The continued presence of initial triggers was not necessary for progressive neurodegeneration}

The contemporary presence of activated microglia and active neurodegeneration in the SN of MPTP-intoxicated patients and monkeys long after clearance of MPTP (Langston et al., 1999; McGeer et al., 2003) implies a continuous crosstalk between microglia and injured neurons during PD progression. However, these in vivo observations cannot determine whether activated microglia are protective, detrimental, or inconsequential during chronic neurodegeneration. We hypothesized that sustained microglial activation (neuroinflammation) drove progressive neurodegeneration, in which persistent existence of initial triggers was not required. We herein examined how the washout of

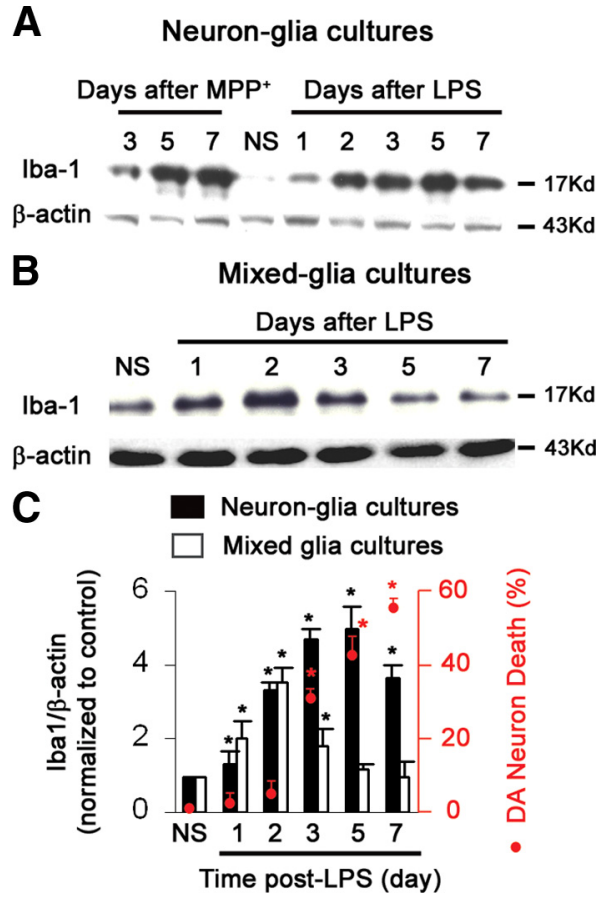

Figure 4. Ongoing neurodegeneration sustained microglial activation. $\boldsymbol{A}-\boldsymbol{C}$, The neuronglia cultures $(\boldsymbol{A}, \boldsymbol{C})$ and mixed-glia cultures ( $\boldsymbol{B}, \boldsymbol{C})$ were treated with normal saline (NS), $0.5 \mu \mathrm{m}$ $\mathrm{MPP}^{+}$, or $10 \mathrm{ng} / \mathrm{ml} \mathrm{LPS}$ for different periods of time and were lysed in RIPA buffer. Cell lysates were size fractionated by 4-12\% SDS-PAGE gels and probed by Western blot for lba1 or $\beta$-actin. $\boldsymbol{C}$, The ratios of densitometry values of Iba 1 and $\beta$-actin in $\boldsymbol{A}$ and $\boldsymbol{B}$ were analyzed and normalized to each responsive control. Quantification of TH-IR neurons in LPS-treated neuronglia cultures revealed time-dependent loss of DA neurons. The long-term upregulation of Iba-1 positively correlated with the neuronal death. The results are the mean \pm SEM of three experiments performed in triplicate. ${ }^{*} p<0.05$ compared with the corresponding saline-treated controls.

LPS and $\mathrm{MPP}^{+}$from the cell cultures affected DA neurodegeneration. $\left[{ }^{3} \mathrm{H}\right] \mathrm{LPS}$ binding and internalization assay indicated that $\sim 90 \%$ of LPS remained in the culture media after the neuronglia cultures were incubated with $10 \mathrm{ng} / \mathrm{ml}$ LPS for $24 \mathrm{~h}$ at $37^{\circ} \mathrm{C}$. The remaining $10 \%$ of LPS either bound to the cell surface of glia (which accounts for $\sim 3 \%$, as indicated by the binding assay conducted at $4^{\circ} \mathrm{C}$ ) or was internalized into glial cells. It is generally agreed that LPS internalization by phagocytes serves to sequester LPS and to limit its interactions with cells.

At 2-24 h after LPS treatment, unbound LPS and released inflammatory factors from activated microglia were removed by washing the neuron-glia cultures. Whereas the wash at 2 or $6 \mathrm{~h}$ attenuated neurotoxicity detected $7 \mathrm{~d}$ after LPS treatment, the wash at 12 or $24 \mathrm{~h}$ did not alter DA neurodegeneration (Fig. $2 A--C)$. The treatment with $10 \mathrm{ng} / \mathrm{ml}$ LPS for 12 or $24 \mathrm{~h}$ in sister culture plates did not cause overt neurotoxicity (Fig. 2D), indicating no existing neuronal loss before the drug washout. Thus, it is conceivable that, once LPS-induced activation of microglia reaches a certain threshold, an activated inflammatory cascade may become propagated even after drug washout and can further damage neurons. Likewise, $2 \mathrm{~d}$ after $\mathrm{MPP}^{+}$treatment, the wash of neuron-glia cultures could not stop DA neurons from additional degeneration (Fig. 2E). These data, combined with our in vivo experimental observation that mice developed delayed and progressive nigral DA neurodegeneration long after clearance of LPS (Qin et al., 2007), imply that neurodegeneration could progress independently of a continuing presence of initial triggers (e.g., LPS and MPP ${ }^{+}$). 
Sustained secretion of inflammatory factors was required for microglial activation-mediated chronic degeneration of DA neurons

As shown in Figure $1 B$, the withdrawal of both activated microglia and their secreted inflammatory/toxic factors $24 \mathrm{~h}$ after LPS treatment provided more protection to DA neurons than the removal of activated microglia alone did. This finding supports an important role of secreted inflammatory factors in microglia-mediated neurodegeneration. Continuing release of superoxide and NO in neuron-glia cultures treated with LPS (Fig. $3 A, B$ ) or $\mathrm{MPP}^{+} / \mathrm{MPTP}$ (Gao et al., 2003a) as well as long-lasting upregulation of gp91 ${ }^{\text {phox }}$ (the catalytic subunit of NADPH oxidase) and iNOS reveal an important role of oxidative damage in persistent inflammation and chronic neurodegeneration (Fig. 3C,D). Moreover, abatement of the production of superoxide and NO in neuron-glia cultures greatly mitigated DA neurodegeneration when diphenyleneiodonium (DPI) and apocynin (NADPH oxidase inhibitors) and 1400W (an iNOS inhibitor) were given $30 \mathrm{~min}$ before and even $48 \mathrm{~h}$ after LPS exposure (Fig. $3 E, F)$. Accordingly, the continued release of NADPH oxidase-derived superoxide and iNOS-derived $\mathrm{NO}$ from activated microglia played a pivotal role in inflammation-mediated progressive DA neurodegeneration.

The interdependency of prolonged microglial activation and chronic, progressive neurodegeneration Given the requirement of microglial activation for progressive DA neurodegeneration (Fig. 1), it is crucial to address how the activation of microglia is maintained. A long-lasting upregulation of Ibal and gradual DA neurodegeneration in neuron-glia cultures treated with $\mathrm{MPP}^{+}$for 3-7 d or LPS for 1-7 d indicate chronic microglial activation during neurodegeneration (Fig. $4 A, C$ ). The upregulation of Ibal in mixed-glia cultures containing astroglia and microglia peaked at $2 \mathrm{~d}$ after LPS treatment and then gradually returned to control level at approximately day 5 (Fig. $4 B, C$ ). The long-term activation of microglia during neurodegeneration was also supported by the persistent secretion of inflammatory/neurotoxic factors and the upregulation of iNOS and gp91 ${ }^{\text {phox }}$ (Fig. 3). These results not only confirm the existence of sustained microglial activation during progressive neurodegeneration but also suggest that ongoing neuronal damage was essential for maintaining chronic neuroinflammation.

\section{HMGB1 bridged persistent neuroinflammation and chronic neurodegeneration}

An array of noxious self-compounds released into the extracellular milieu by injured neurons can induce microglial activation (reactive microgliosis). As introduced earlier, HMGB1 can be
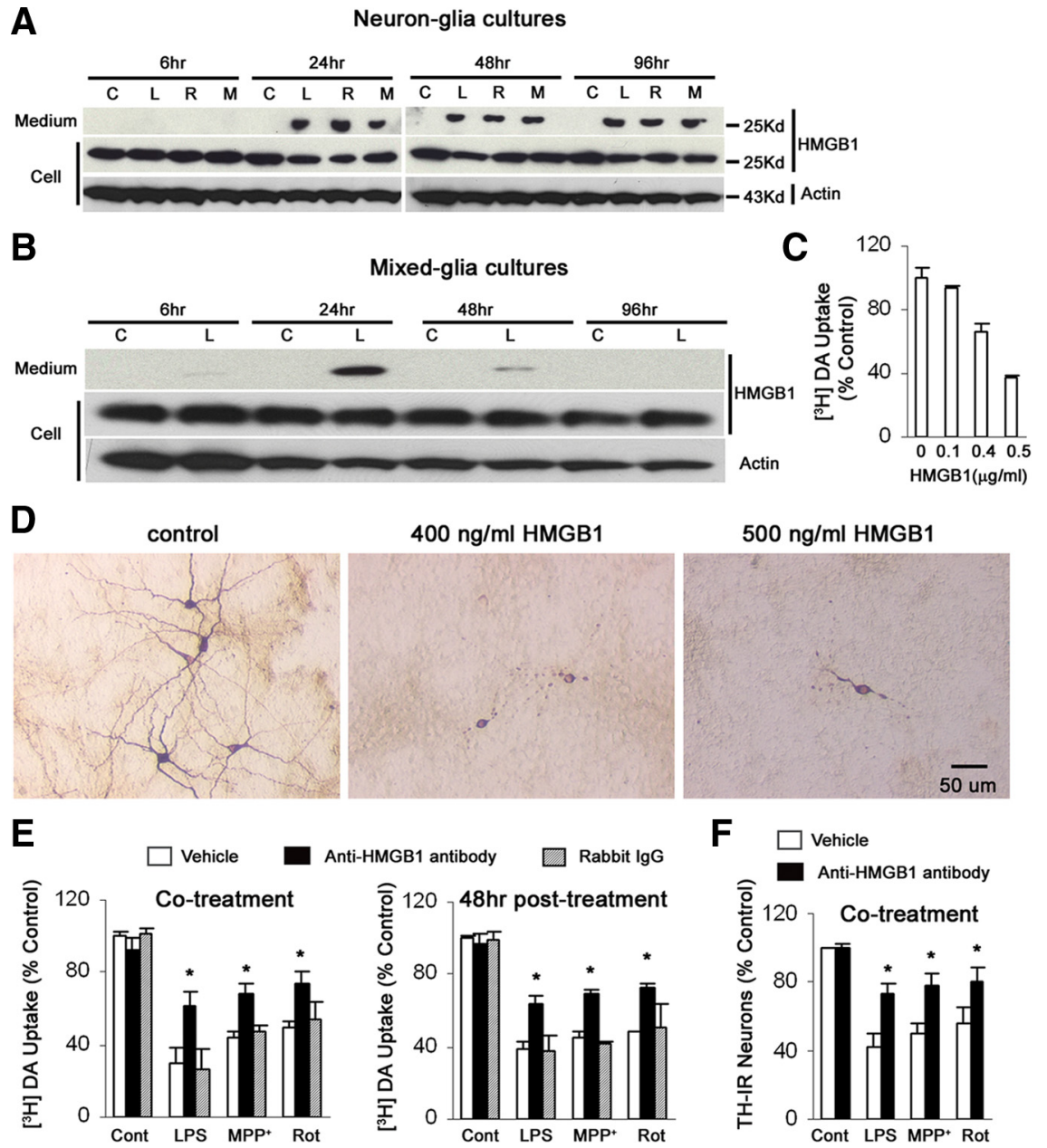

$500 \mathrm{ng} / \mathrm{ml}$ HMGB1

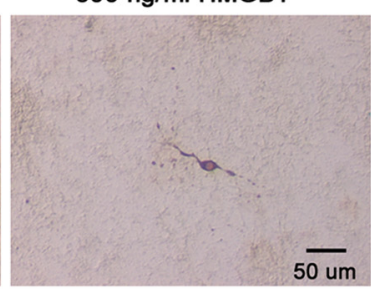

F $\square$ vehicle

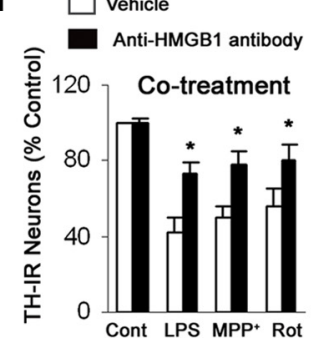

Figure 5. HMGB1 mediated persistent crosstalk between activated microglia and degenerating neurons. $A, B$, The neuron- glia cultures $(\boldsymbol{A})$ and mixed-glia cultures $(\boldsymbol{B})$ were treated with normal saline (NS), $10 \mathrm{ng} / \mathrm{ml} \mathrm{LPS}, 10 \mathrm{~nm}$ rotenone, or $0.5 \mu \mathrm{M} \mathrm{MPP}{ }^{+}$. The

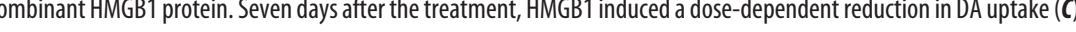
Neutralization of HMGB1 rescued DA neurons from chronic degeneration. During or $48 \mathrm{~h}$ after the addition of $10 \mathrm{ng} / \mathrm{ml} \mathrm{LPS}, 0.5 \mu \mathrm{M}$ $\mathrm{MPP}^{+}$, or $10 \mathrm{~nm}$ rotenone, neuron- glia cultures were treated with rabbit lgG isotype antibody or anti-HMGB1 antibody. Seven days after the initial treatment, cultures were assayed for DA uptake $(\boldsymbol{E})$ and cell count of TH-IR neurons $(\boldsymbol{F})$. The results are the mean \pm SEM of three to four experiments performed in triplicate. ${ }^{*} p<0.05$ compared with the corresponding LPS-, MPP $^{+}-$, or rotenone-treated cultures. C, Control; L, LPS; R, rotenone; $M, M P P{ }^{+}$.

passively released from necrotic cells or actively secreted from inflammatory cells to initiate inflammatory responses. We next examine how extracellular cytokine-like effects of HMGB1 affected chronic and progressive dopaminergic neurodegeneration, which has not been studied before. As seen in Figure $5 A$, HMGB1 was recovered in culture media after the neuron-glia cultures were treated with LPS, MPP ${ }^{+}$, or rotenone for $24-96 \mathrm{~h}$. The potential source of such HMGB1-activated microglia and/or dying/dead neurons_ - could vary depending on the toxin and the time of measurement.

In LPS-treated mixed-glia cultures, the release of HMGB1 was detectable at $6 \mathrm{~h}$, reached peak levels at $24 \mathrm{~h}$, and was untraceable at $96 \mathrm{~h}$ by Western blotting analysis (Fig. $5 B$ ). The long-lasting release of HMGB1 in treated neuron-glia cultures, but not mixed-glia cultures, prompts us to reason that HMGB1 could be an important mediator derived from degenerating neurons to maintain prolonged inflammation and to subsequently induce 
A
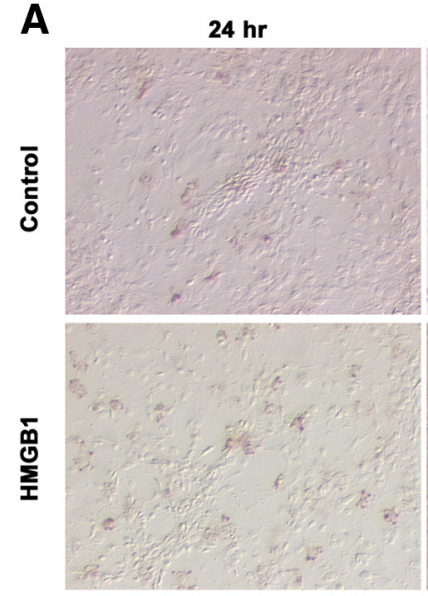

B

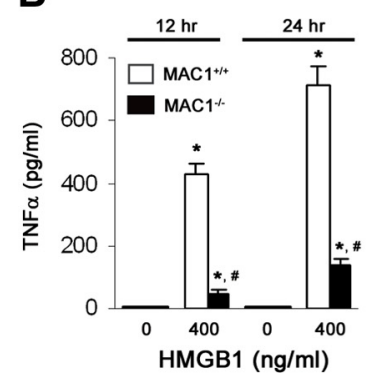

$48 \mathrm{hr}$

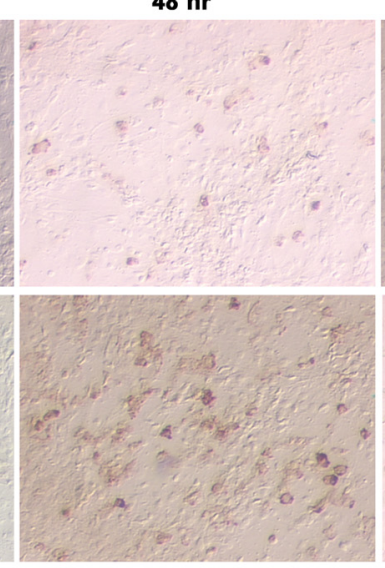

C

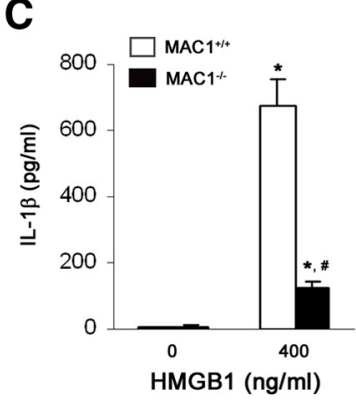

$96 \mathrm{hr}$

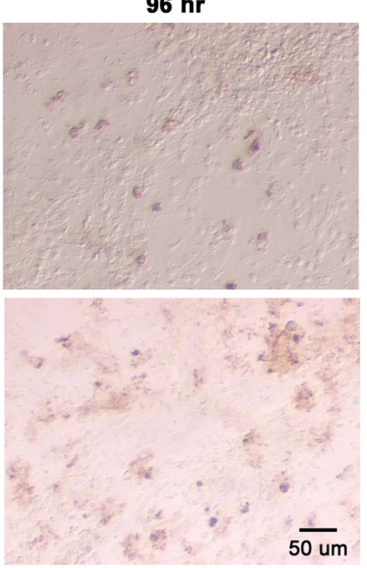

D

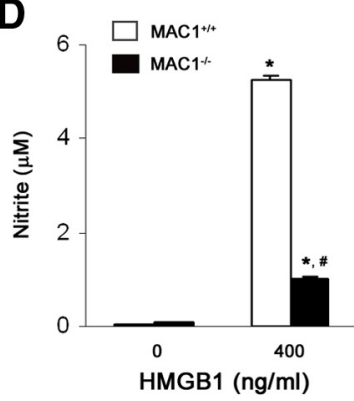

Figure 6. HMGB1 activated microglia and the deficiency in microglial Mac1 attenuated HMGB1-mediated release of inflammatory mediators. A, Neuron-glia cultures were treated for 1- $4 \mathrm{~d}$ with the vehicle or $400 \mathrm{ng} / \mathrm{ml} \mathrm{HMGB1}$ and then were immunostained with the 0X-42 antibody. In control cultures, OX-42-IR microglia were small and round. After stimulation with HMGB1, microglia revealed increased immunoreactivity and became larger in size and irregular in shape, indicative of activation. $\boldsymbol{B}-\boldsymbol{D}$, Neuron-glia cultures prepared from wild-type or $\mathrm{MaC1}^{-/-}$mice were treated with vehicle or $400 \mathrm{ng} / \mathrm{ml} \mathrm{HMGB1}$ for 12 or $24 \mathrm{~h}$. Levels of TNF- $\alpha(\boldsymbol{B})$, IL-1 $\beta(24 \mathrm{~h})(\boldsymbol{C})$, and nitrite (an indicator of N0 production, $24 \mathrm{~h})(\boldsymbol{D})$ in the culture medium were measured. Results are means \pm SEM of three experiments performed in triplicate. ${ }^{*} p<0.05$ compared with the corresponding vehicletreated control cultures. ${ }^{\#} p<0.05$ compared with the corresponding HMGB1-treated wild-type cultures.

chronic neurodegeneration. In support of this hypothesis, endotoxin-free recombinant HMGB1 protein $(400 \mathrm{ng} / \mathrm{ml})$ caused chronic dopaminergic neurodegeneration in neuron-glia cultures (Fig. 5C,D). Furthermore, anti-HMGB1 neutralizing antibody attenuated dopaminergic neurodegeneration, when given simultaneously with or $48 \mathrm{~h}$ after $\mathrm{MPP}^{+}$, rotenone, or LPS (Fig. $5 E, F)$. In addition, after stimulation with HMGB1 for 24-96 h, microglia exhibited larger cell bodies, irregular shape, and increased immunoreactivity to OX-42 antibody, which indicated microglial activation (Fig. 6A). HMGB1 also stimulated microglia to release inflammatory and neurotoxic factors, including TNF- $\alpha$, IL- $1 \beta$, and NO (Fig. $6 B-D$ ).

\section{Interaction between HMGB1 and microglial Macl in mediating chronic inflammation and progressive neurodegeneration}

HMGB1 has been reported to signal through multi-ligand receptors, including RAGE (receptor for advanced glycation end products), toll-like receptor 2 (TLR2), and TLR4 in mediating inflammatory reactions. The structural conformation of HMGB1 allows it to function as a damage-associated molecular pattern molecule (DAMP) (Seong and Matzinger, 2004). DAMPs trigger pattern recognition receptors (PRRs) and signal to the immune system of tissue injury to elicit a noninfectious inflammatory response (Dumitriu et al., 2005; Tian et al., 2009). Macl belongs to the $\beta 2$ integrin family and also functions as a PRR (Fan and Edgington, 1993; Ross and Větvicka, 1993; Ross, 2000). We there- fore proposed that HMGB1 interacts with microglial Mac1 and thereby mediates chronic neuroinflammation and progressive neurodegeneration.

To test this hypothesis, various primary cell cultures were generated from Mac1deficient mice. During HMGB1 stimulation, $\mathrm{MaCl}^{-/-}$microglia produced much less TNF- $\alpha$, IL- $1 \beta$, and NO (Fig. $6 B-D$ ). Mac1 deficiency also alleviated HMGB1mediated chronic neurodegeneration, as shown by the attenuated dendrite degeneration as well as less reduction in DA uptake and in the number of TH-IR neurons in $\mathrm{Mac1}^{-/-}$neuron-glia cultures compared with wild-type cultures. HMGB1 at 400 $\mathrm{ng} / \mathrm{ml}$ concentration did not show neurotoxicity in neuron-enriched cultures (Fig. $7 A, B)$. These data together elucidate that HMGB1 induced chronic neuroinflammation and caused progressive neurodegeneration, which may be mediated by its interaction with Macl. The findings that deletion of microglial Macl greatly promoted the survival of DA neurons after the cultures were treated for $7 \mathrm{~d}$ with LPS and rotenone (Fig. 7C,D) or with $\mathrm{MPP}^{+}$ (Hu et al., 2008) revealed an important role of Mac1 in chronic neuroinflammation and neurodegeneration.

Next, we seek direct evidence of interactions between HMGB1 and Mac1. Microglia, prepared from rats and $\mathrm{Macl}^{-/-}$ and wild-type mice were treated with vehicle or $500 \mathrm{ng} / \mathrm{ml} \mathrm{HMGB1}$ at $4^{\circ} \mathrm{C}$ for $2 \mathrm{~h}$ or $37^{\circ} \mathrm{C}$ for $15 \mathrm{~min}$. Coimmunoprecipitation experiments indicated that the anti-HMGB1 antibody coprecipitated Mac1 in HMGB1-treated wild-type microglia but not $\mathrm{Macl}^{-/-}$microglia. Here, the same immunoprecipitation samples were blotted and probed with anti-Macl antibody, stripped, and then reprobed with an antibody against HMGB1. Conversely, anti-Macl antibody coprecipitated HMGB1 in $\mathrm{Macl}^{+/+}$microglia but not $\mathrm{Macl}^{-/-}$microglia (Fig. $8 \mathrm{~A}$ ). In contrast, the immunoprecipitation using an IgG isotype control antibody was negative for both HMGB1 and Macl (data not shown). The binding analysis of microglial membrane fractions with purified recombinant $\mathrm{HMGB} 1$ protein $(1 \mu \mathrm{g} / \mathrm{ml})$ at $4^{\circ} \mathrm{C}$ for $3 \mathrm{~h}$ was used to further validate the physical interaction between HMGB1 and Mac1 (Fig. 8B). After the binding incubation and extensive washes by high-speed sedimentation, membrane fractions were solubilized in sample buffer. The following immunoblotting revealed that more HMGB1 bound to $\mathrm{Macl}^{+/+}$ microglial membranes than bound to $\mathrm{Macl}^{-/-}$microglial membranes. When both membrane fractions were incubated with vehicle, no HMGB1 was detected, indicating the specificity of this binding analysis. The binding results further implicate Macl as a potential receptor for HMGB1 on microglia.

\section{The binding of HMGB1 to Macl activated microglial nuclear factor- $\kappa \mathrm{B}$ pathway}

As mentioned above, extracellular HMGB1 can bind RAGE, TLR2, and TLR4 and signal via the nuclear factor $-\kappa \mathrm{B}(\mathrm{NF}-\kappa \mathrm{B})$ pathway. We first evaluated the involvement of NF- $\kappa$ B path- 
way in Mac1-mediated cytokine-like effects of HMGB1. The activation of NF- $\kappa \mathrm{B}$ is tightly regulated by the $\mathrm{I} \kappa \mathrm{B}$ kinase (IKK) complex composed of catalytic subunits, IKK $\alpha$ and IKK $\beta$, and a regulatory subunit, IKK $\gamma$. In response to inflammatory stimuli, $\mathrm{I} \kappa \mathrm{Bs}$ are rapidly phosphorylated by IKK complex and degraded by the proteasome pathway. Loss of $\mathrm{I} \kappa \mathrm{B}$ allows the release and the nuclear translocation of p50/p65 NF- $\kappa \mathrm{B}$ dimers, regulating the expression of target genes (Zhang and Ghosh, 2001; Hoffmann et al., 2002).

After microglia were treated with HMGB1 for 15 or $30 \mathrm{~min}$, IKK $\alpha / \beta$ phosphorylation could be detected in wildtype microglia but not $\mathrm{Macl}^{-/-}$microglia (Fig. 8C,D). In $\mathrm{Macl}^{+/+}$microglia, I $\kappa \mathrm{B} \alpha$ was phosphorylated and sequentially degraded after HMGB1 treatment. Unlike wild-type microglia, Mac1-null microglia did not show much degradation of $\mathrm{I} \kappa \mathrm{B} \alpha$. HMGB1 treatment caused strong p65 phosphorylation in wild-type microglia. Lower level of p65 phosphorylation was also detectable in $\mathrm{Macl}^{-/-}$microglia 15 min after HMGB1 addition (Fig. 8C,D), which may result from the interaction between HMGB1 and TLR2, TLR4, or RAGE. It is also possible that HMGB1 might activate alternative NF- $\kappa \mathrm{B}$ pathways, because p50/p65 can be phosphorylated by multiple kinases (e.g., protein kinase A). p65 phosphorylation regulates activation, nuclear localization, proteinprotein interactions, and transcriptional activity. Indeed, nuclear translocation of p65 was observed in rat microglia treated with HMGB1 (Fig. 8E,F). Difference in NF- $\kappa \mathrm{B}$ target gene products (e.g., TNF- $\alpha$, IL-1 $\beta$, and NO) in $\mathrm{Macl}^{-/-}$and $\mathrm{Macl}^{+/+}$ microglia after HMGB1 treatment (Fig. 6B-D) was consistent with the differential NF- $\kappa \mathrm{B}$ activation in these microglia (Fig. $8 C, D)$. Thus, Mac1/HMGB1 interaction induced the activation of microglial NF- $\kappa \mathrm{B}$ pathway and mediated neuroinflammation.

\section{HMGB1-mediated activation of NADPH oxidase required Mac1}

Multiple lines of evidence have documented a pivotal role of overactivated NADPH oxidase in inflammation-mediated neurodegeneration. Furthermore, recent evidence has indicated a critical participation of Macl in the activation of NADPH oxidase during inflammation (Coxon et al., 1996; Lynch et al., 1999; Le Cabec et al., 2002; Gao and Hong, 2008). We, therefore, investigated the involvement of NADPH oxidase and its upstream signaling pathway in HMGB1-mediated chronic microglial activation. The results showed that the treatment of primary microglia cultures with $500 \mathrm{ng} / \mathrm{ml}$ HMGB1 resulted in membrane translocation of $\mathrm{p} 47^{\text {phox }}$, a cytosolic subunit of NADPH oxidase. The deficiency in Macl prevented such translocation (Fig. 9A,B). In addition, during stimulation with HMGB1, only wild-type microglia, but not $\mathrm{Macl}^{-/-}$or $g p 91^{\text {phox-/- }}$ microglia, released

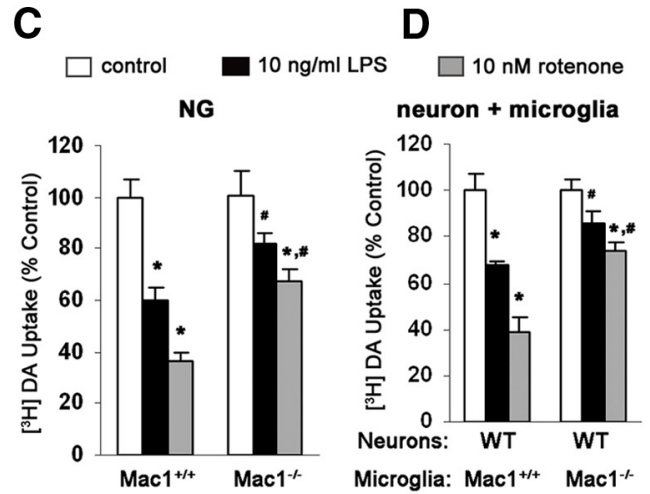

$400 \mathrm{ng} / \mathrm{ml}$ HMGB1

$500 \mathrm{ng} / \mathrm{ml}$ HMGB1

Figure 7. Chronic DA neurodegeneration was attenuated in the absence of microglial Mac1. Neuron-glia cultures $(\boldsymbol{A}, \boldsymbol{B})$ and (the neuron- glia cultures immunostained for TH (from 3 experiments) displayed greater lesions of DA of microglial Mac1 rendered DA neurons more resistant to inflammation-mediated neurotoxicity. All quantification results are means \pm SEM of three to four experiments performed in triplicate. NG, Neuron-glia cultures; $N$, neuron-enriched cultures.

extracellular superoxide; cotreatment of these microglial cells with DPI and apocynin prevented the superoxide release (Fig. 9C). These findings indicated that $\mathrm{Macl}$ was required for HMGB1-mediated activation of NADPH oxidase. The resistance of neuron-glia cultures deficient in NADPH oxidase $\left(g p 91^{\text {phox-/- }}\right)$ to HMGB1-mediated neurotoxicity (Fig. 9D) further emphasized the important role of NADPH oxidase in chronic neuroinflammation and neurodegeneration. In summary, our findings demonstrated that HMGB1/Mac1 interaction induced the activation of microglial NF- $\kappa \mathrm{B}$ pathway and NADPH oxidase enzyme, thereby mediated persistent neuroinflammation and progressive neurodegeneration.

\section{Discussion}

The present study demonstrates that an uncontrolled, prolonged inflammatory process may be a driving force for the progressive degeneration of DA neurons in PD. Microglia were indispensable for the chronic DA neurodegeneration induced by neurotoxins $\left(\mathrm{MPP}^{+}\right.$and rotenone) or inflammogen LPS. Through a vicious cycle formed between injured neurons and unregulated neuroinflammation, the neurodegeneration continued to progress even 
A

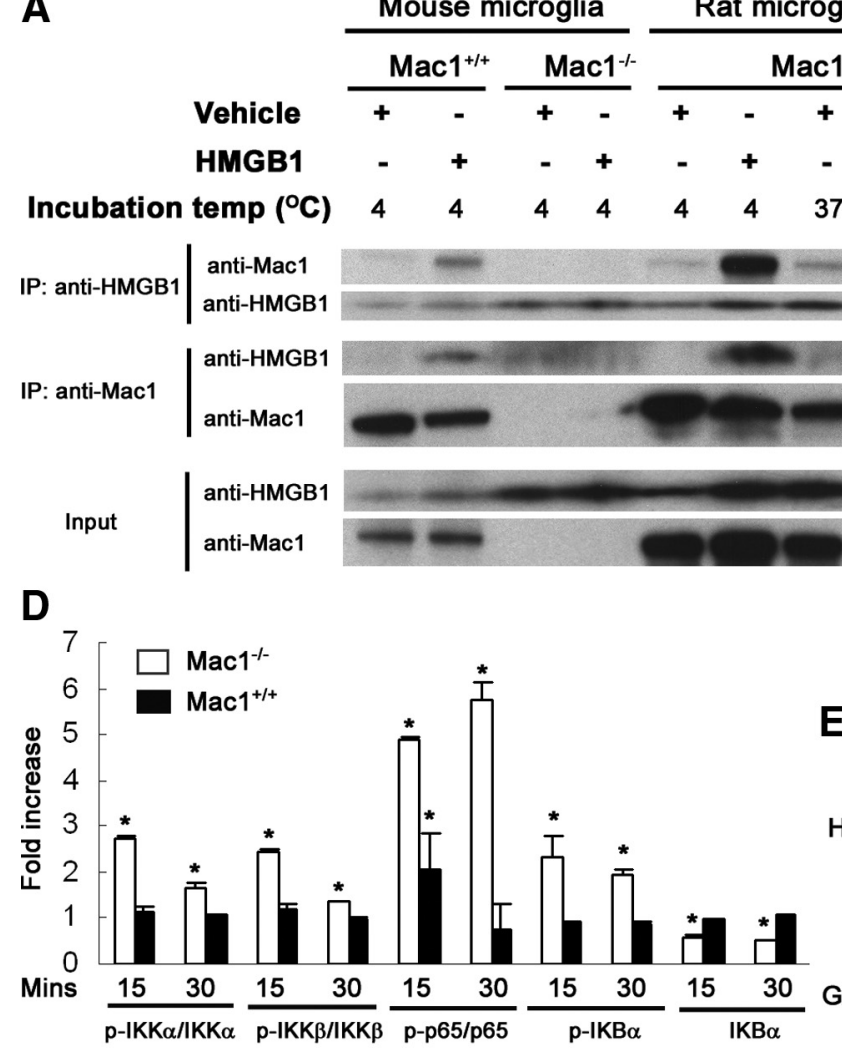

B

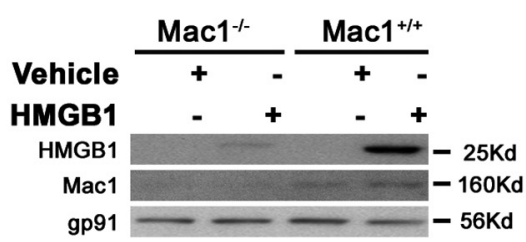

C

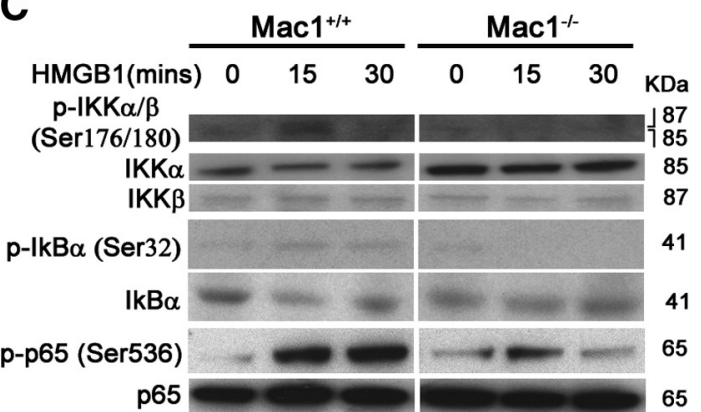

E

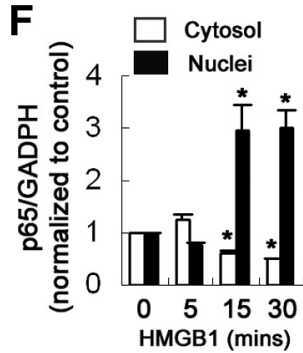

Figure 8. Interactions between HMGB1 and Mac1. A, Primary microglia from rat, $M a c 1^{-/-}$mice, or wild-type mice were treated with vehicle or $500 \mathrm{ng} / \mathrm{ml} \mathrm{HMGB} 1$ at $4^{\circ} \mathrm{C}$ for $2 \mathrm{~h}$ or $37^{\circ} \mathrm{C}$ for 15 min. Cell lysates were collected in TNE buffer and then immunoprecipitated with antibodies against HMGB1 or Mac1. Separated immunoprecipitates were immunoblotted and probed for Mac1 and, after stripping the membrane, for HMGB1 or vice versa. Anti-HMGB1 antibody coimmunoprecipitated Mac1 in wild-type microglia treated with HMGB1, whereas anti-Mac1 antibody coimmunoprecipitated HMGB1. IP, Immunoprecipitation. $B$, Crude membrane fractions from Mac $1^{-/-}$or Mac $1^{+/+}$microglia were incubated with $1 \mu \mathrm{g} / \mathrm{ml} \mathrm{HMGB1}$ or vehicle at $4^{\circ} \mathrm{C}$ for $3 \mathrm{~h}$. The Western blotting indicated more HMGB1 bound to $\mathrm{MaC1}^{+/+}$microglial membranes than bound to Mac1 ${ }^{-/-}$microglial membranes. $\mathrm{C}$, Primary microglia from Mac $1^{-/-}$or wild-type mice were treated with vehicle or $500 \mathrm{ng} / \mathrm{ml} \mathrm{HMGB} 1$ at $37^{\circ} \mathrm{C}$ for 15 or $30 \mathrm{~min}$. Whole-cell lysates were extracted in RIPA buffer. The activation of NF- $\kappa B$ pathway, as shown by phosphorylation of IKK $\alpha / \beta$, degradation of $I_{\kappa B}$, and phosphorylation of p65, was observed in HMGB1-treated wild-type microglia, but such activation was blunted in HMGB1-treated Mac $1^{-/-}$microglia. $\boldsymbol{D}$, The ratios of densitometry values of examined proteins in $\boldsymbol{C}$ were normalized to each responsive control. ${ }^{*} p<0.05$ compared with the corresponding vehicle-treated controls. $\boldsymbol{E}, \boldsymbol{F}$, Nuclear translocation of p65 was observed in rat microglia treated with HMGB1 (500 ng/ml) for 15-30 min. GAPDH, Glyceraldehyde-3-phosphate dehydrogenase. All panels are representative of two to three independent experiments.

after the initial toxic triggers (LPS and $\mathrm{MPP}^{+}$) were withdrawn. Sustained release of reactive free radicals from activated microglia and HMGB1 from dying/dead neurons and activated microglia was critical for the formation and the maintenance of this vicious cycle. Additional mechanistic studies indicate that interactions between HMGB1 and microglial Mac1 bridged persistent neuroinflammation and progressive dopaminergic neurodegeneration. This study pinpoints major mediators linking persistent inflammation and chronic dopaminergic neurodegeneration, which may facilitate our understanding of the mechanism of the progressive nature of $\mathrm{PD}$.

The involvement of neuroinflammation in PD and other neurodegenerative diseases has long been recognized (Kreutzberg, 1996). The pioneer work of Dr. McGeer and his colleagues reveals reactive microglia in the SN of Parkinson's and Alzheimer's disease brains (McGeer et al., 1988). Since then, numerous studies have reported elevated levels of inflammatory mediators in the brain of various neurodegenerative diseases (Yamada et al., 1992; Mogi et al., 1994; Liu et al., 2003). Nevertheless, these observations left the question open whether the observed neuroinflammation is beneficial, detrimental, or inconsequential to neuronal loss. Recently, neuroinflammation is increasingly accepted as a double-edged sword (Nguyen et al., 2002; Wyss-Coray and Mucke, 2002). Microglia can remove toxic proteins (e.g., amyloid plaques), clear cell debris, secret neurotrophic factors, and prevent neurodegeneration (Schwartz, 2002; Simard et al., 2006). Conversely, ample evidence points to detrimental roles of neuroinflammation in neurodegenerative diseases (Liberatore et al., 1999; Du et al., 2001; Gayle et al., 2002; Glass et al., 2010; Przedborski, 2010).

Here, we provided direct experimental evidence implicating persistent microglial activation as the major driving cause of progressive neurodegeneration in PD. This conclusion is supported by the following experimental findings: first, dopaminergic neurodegeneration progressed markedly over time in neuron-glia cultures treated with $\mathrm{MPP}^{+}$, rotenone, or LPS but not in neuronenriched cultures treated with the same toxins (Fig. 1A); second, withdrawal of activated microglia and released inflammatory mediators prevented DA neurons from LPS-induced progressive degeneration (Fig. $1 B$ ); third, the continued presence of initial triggers (e.g., LPS and $\mathrm{MPP}^{+}$) was not required for persistent microglial activation and progressive neurodegeneration (Fig. 2); fourth, continuing release of superoxide and NO from activated microglia in neuron-glia cultures treated with LPS (Fig. 3) or

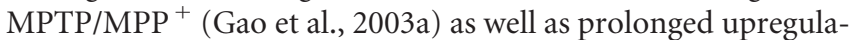
tion of inflammatory enzymes (NADPH oxidase and iNOS) in LPS-treated neuron-glia cultures (Fig. 3) pointed to a critical role of inflammation-elicited oxidative insults in chronic neuro- 
A
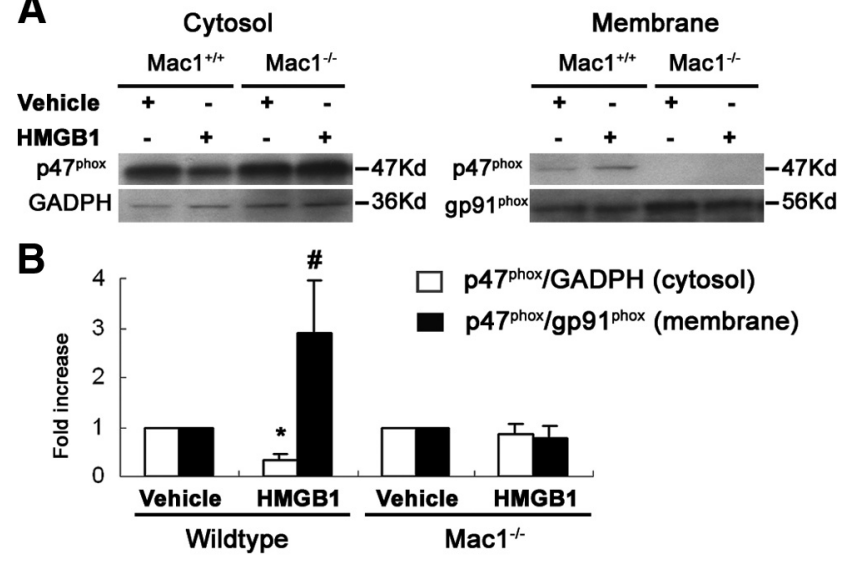

C
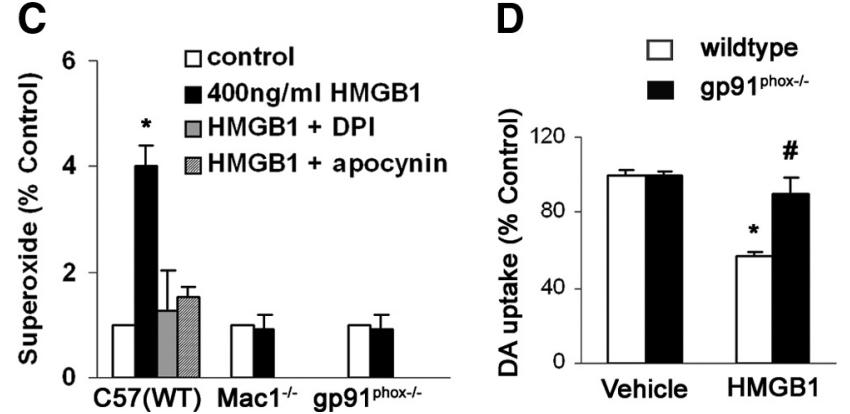

Figure 9. HMGB1 activated the Mac1-NADPH oxidase signaling axis leading to microglial activation. $A$, Mac1 was required for HMGB1-mediated $\mathrm{p} 47^{\text {phox }}$ translocation to cell membrane. Primary microglia-enriched cultures prepared from $\mathrm{MaC1}^{-1-}$ mice or wild-type mice were treated with vehicle or $500 \mathrm{ng} / \mathrm{ml} \mathrm{HMGB} 1$ at $37^{\circ} \mathrm{C}$ for $15 \mathrm{~min}$. Subcellular fractions were isolated to perform Western blotting analysis for $\mathrm{p} 47^{\text {phox }}$ levels in membrane and cytosolic fractions of microglia. Glyceraldehyde-3-phosphate dehydrogenase (GADPH) and gp $91^{\text {phox }}$ were used as internal control of cytosolic and membrane fractions, respectively. $\boldsymbol{B}$, The ratio of densitometry values of cytosolic $p 47^{\text {phox }}$ to GADPH and membrane $\mathrm{p} 47^{\text {phox }}$ to gp9 ${ }^{\text {phox }}$ from two independent experiments was normalized to each respective vehicle-treated control. C, Primary micro-

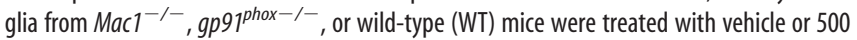
$\mathrm{ng} / \mathrm{ml} \mathrm{HMGB1}$ at $37^{\circ} \mathrm{C}$ for $15 \mathrm{~min}$. The production of extracellular superoxide was detected by SOD-inhibitable reduction of WST-1. The production of extracellular superoxide was detected only in HMGB1-treated wild-type microglia and was inhibited by the cotreatment of the cultures with DPI and apocynin; microglia with deficiency in Mac1 or gp91 ${ }^{\text {phox }}$ did not cause detectable superoxide release after HMGB1 treatment. $\boldsymbol{D}$, Primary neuron-glia cultures from gp $91^{\text {phox-1- }}$ or wild-type mice were stimulated with $400 \mathrm{ng} / \mathrm{ml} \mathrm{HMGB1}$. Seven days later, the neurotoxicity was evaluated by $\left[{ }^{3} \mathrm{H}\right] \mathrm{DA}$ uptake assay. Results were expressed as a percentage of the vehicle-treated control cultures and were the mean \pm SEM from three to four independent experiments. ${ }^{*} p<0.05$ compared with respective vehicle-treated control cultures. ${ }^{\#} p<0.05$ compared with HMGB1-treated wild-type cultures.

degeneration; fifth, posttreatment with inhibitors against NADPH oxidase and iNOS significantly attenuated LPS-mediated progressive neurodegeneration (Fig. 3); finally, microglial activation induced by chronic infusion of LPS into the SN or by a single systemic LPS injection mediated delayed and progressive degeneration of nigral DA neurons in rodents (Gao et al., 2002b; Ling et al., 2006; Qin et al., 2007). Together, these results lend strong credence to the concept that uncontrolled microglial activation, either as an initiator or as a secondary contributor, could drive a chronic, progressive neurodegenerative process.

The positive feedback between activated microglia and damaged neurons is crucial for both chronic neuroinflammation and progressive neurodegeneration. Acute insults to the CNS such as environmental neurotoxins can directly trigger immediate neuronal lesions. Injured neurons activate microglia through generating a spectrum of noxious endogenous substances in the extracellular milieu. Once activated, microglia secret various inflammatory and neurotoxic factors (e.g., cytokines, reactive free radicals, and proteases) and exacerbate ongoing neurodegeneration (Kreutzberg, 1996; Liu et al., 2003; Block et al., 2007). Thus, no matter which comes first, neurodegeneration and neuroinflammation, once reaching a certain threshold, propagate to form a vicious cycle (Gao and Hong, 2008). This cycle not only further augments the ongoing pathology but also makes neurodegeneration become a chronic, progressive process. Therefore, it is the activation of microglia that enables an initial acute neuronal injury to transform into chronic and progressive neurodegeneration. Chronic neuroinflammation and progressive neurodegeneration may become integrated and inseparable in mediating chronic PD progression.

DAMPs (e.g., ATP, heat-shock proteins, S100 proteins, and HMGB1) naturally are nuclear or cytosolic molecules with defined intracellular function. When released extracellularly, DAMPs can activate microglia. HMGB1 is reported to link acute neuron necrosis and delayed neuroinflammation in ischemic brain damage (Kim et al., 2006; Muhammad et al., 2008). Additionally, diffuse deposits of HMGB1 are found around dying neurons in rat models of Alzheimer's disease (Takata et al., 2004). In the present study, we found that HMGB1 not only stimulated microglia to release inflammatory factors, including TNF $\alpha$, IL$1 \beta$, and NO (Fig. 6), but also caused chronic dopaminergic neurodegeneration in the presence of microglia (Fig. 5C,D). More importantly, the long-term release of HMGB1 in neuron-glia cultures treated with $\mathrm{MPP}^{+} / \mathrm{LPS} /$ rotenone but not in LPStreated mixed-glia cultures, along with the neuroprotective effect of the neutralization of HMGB1 (Fig. 5), suggests that HMGB1 from degenerating neurons may be an important mediator linking sustained neuroinflammation to progressive dopaminergic neurodegeneration in PD.

The involvement of multiple separate receptors (e.g., TLR2, TLR4, and RAGE) in HMGB1-mediated cellular and biological responses has been well described (Scaffidi et al., 2002; Lotze and Tracey, 2005). Interestingly, although recent findings bring more attention to the HMGB1-TLR4 pathway (Maroso et al., 2010; Mittal et al., 2010), HMGB1 has also been reported to cause lethality in TLR4-defective $\mathrm{C} 3 \mathrm{H} / \mathrm{HeJ}$ mice, which points to a TLR4-independent pathway for HMGB1 (Sims et al., 2010). There is a great deal of interest in defining novel receptors and binding partners for HMGB1 and in elucidating the signal transduction mechanisms underlying the HMGB1 receptor-ligand interactions. Here, we identified microglial Macl as a receptor of HMGB1. Mac1 (also known as complement receptor 3, CD11b/ CD18, or $\alpha_{\mathrm{M}} \beta_{2}$ ) is an adhesion molecule and also functions as a PRR (Wright and Jong, 1986; Ross and Vĕtvicka, 1993; Ross, 2000; Pei et al., 2007; Zhang et al., 2007). PRRs including Mac1 expressed broadly on microglia can react to DAMPs and other aberrant endogenous ligands in neuronal tissues (Floden et al., 2005; Block and Hong, 2007). In the brain, Macl is involved in microglial adhesion, chemotaxis, phagocytosis, and activation (Mayadas and Cullere, 2005). The expression of Mac1 is elevated in PD brains (Liberatore et al., 1999). Mac1 deficiency in microglia greatly attenuated chronic impairment of DA neurons induced by LPS, rotenone (Fig. 7), or MPTP (Hu et al., 2008). Because neither MPTP nor $\mathrm{MPP}^{+}$can directly activate microglia (Gao et al., 2003a), Mac1 seems to be a target for some of the noxious endogenous compounds generated after neuronal injuries. In support of this notion, Mac1 was found to be required for the microglia-mediated enhanced neurotoxicity induced by $\alpha$-synuclein in neuron-glia cultures (Zhang et al., 2007). 
$\alpha$-Synuclein is predominantly an intracellular neuronal protein, but it has also been recovered from human blood and CSF (ElAgnaf et al., 2003; Hong et al., 2010). Similarly, $\beta$-amyloid ( $\beta 25-$ 35 ) has been reported to bind to Macl and to mediate microglial release of NO (Goodwin et al., 1997).

We demonstrated in the present study that HMGB1 interacted with microglial Macl and this interaction mediated persistent neuroinflammation and consequent progressive neurodegeneration. Specifically, the coimmunoprecipitation and the binding analysis revealed the physical interaction between HMGB1 and Mac1 (Fig. $8 A, B$ ). Consistent with this physical interaction, functional assays indicated that the binding of HMGB1 to microglial Mac1 activated microglia NF- $\kappa \mathrm{B}$ pathway and NADPH oxidase. The activation of both NF- $\kappa \mathrm{B}$ and NADPH oxidase was blunted in $\mathrm{Macl}^{-1-}$ microglia (Figs. $8 \mathrm{C}-\mathrm{F}, 9 \mathrm{~A}-\mathrm{C}$ ). As a result, $\mathrm{Macl}^{-1-}$ microglia released less inflammatory factors and superoxide during HMGB1 stimulation and the deficiency of microglial Mac1 and NADPH oxidase attenuated HMGB1-mediated neurodegeneration (Figs. $6 B-D, 7,9$ ). The HMGB1 was able to induce membrane translocation of $\mathrm{p} 47^{\text {phox }}$ in microglia, which required the presence of Mac1 (Fig. 9A). Thus, the coupling between Mac1 and NADPH oxidase might be an important mechanism by which HMGB1 mediated chronic microglial activation and induced progressive neurodegeneration.

The deleterious effects of excessive microglial activation are not limited to PD. More recent evidence suggests that an inflammatory mechanism is commonly shared by a large group of neurodegenerative disorders (McGeer and McGeer, 2003; Gao and Hong, 2008; Glass et al., 2010). For instance, the progression, rather than the onset, of amyotrophic lateral sclerosis (a progressive paralytic neurodegenerative disorder) is affected by the expression of mutant superoxide dismutase in microglia (Boillée et al., 2006). With awareness of a driving role of overactivation of microglia in neurodegenerative diseases, pharmacological targeting of secondary neurodegeneration mediated by uncontrolled microglial activation could be of therapeutic value for slowing down the disease progression. In conclusion, our data together provide direct experimental evidence indicating that uncontrolled, prolonged inflammation may be a driving force of progressive dopaminergic neurodegeneration in $\mathrm{PD}$. Interactions between HMGB1 (derived from degenerating neurons and activated microglia) and microglial Macl sustained chronic inflammation and mediated chronic dopaminergic neurodegeneration. Mac1 might become a promising target for the development of therapeutic agents halting the vicious cycle between uncontrolled neuroinflammation and degenerating neurons and thereby retarding the progression of PD.

\section{References}

Betarbet R, Sherer TB, MacKenzie G, Garcia-Osuna M, Panov AV, Greenamyre JT (2000) Chronic systemic pesticide exposure reproduces features of Parkinson's disease. Nat Neurosci 3:1301-1306.

Block ML, Hong JS (2007) Chronic microglial activation and progressive dopaminergic neurotoxicity. Biochem Soc Trans 35:1127-1132.

Block ML, Zecca L, Hong JS (2007) Microglia-mediated neurotoxicity: uncovering the molecular mechanisms. Nat Rev Neurosci 8:57-69.

Boillée S, Yamanaka K, Lobsiger CS, Copeland NG, Jenkins NA, Kassiotis G, Kollias G, Cleveland DW (2006) Onset and progression in inherited ALS determined by motor neurons and microglia. Science 312: 1389-1392.

Coxon A, Rieu P, Barkalow FJ, Askari S, Sharpe AH, von Andrian UH, Arnaout MA, Mayadas TN (1996) A novel role for the beta 2 integrin CD11b/CD18 in neutrophil apoptosis: a homeostatic mechanism in inflammation. Immunity 5:653-666.

Du Y, Ma Z, Lin S, Dodel RC, Gao F, Bales KR, Triarhou LC, Chernet E, Perry
KW, Nelson DL, Luecke S, Phebus LA, Bymaster FP, Paul SM (2001) Minocycline prevents nigrostriatal dopaminergic neurodegeneration in the MPTP model of Parkinson's disease. Proc Natl Acad Sci U S A 98:14669-14674.

Dumitriu IE, Baruah P, Manfredi AA, Bianchi ME, Rovere-Querini P (2005) HMGB1: guiding immunity from within. Trends Immunol 26:381-387.

El-Agnaf OM, Salem SA, Paleologou KE, Cooper LJ, Fullwood NJ, Gibson MJ, Curran MD, Court JA, Mann DM, Ikeda S, Cookson MR, Hardy J, Allsop D (2003) Alpha-synuclein implicated in Parkinson's disease is present in extracellular biological fluids, including human plasma. FASEB J 17:1945-1947.

Fan ST, Edgington TS (1993) Integrin regulation of leukocyte inflammatory functions. CD11b/CD18 enhancement of the tumor necrosis factor-alpha responses of monocytes. J Immunol 150:2972-2980.

Floden AM, Li S, Combs CK (2005) Beta-amyloid-stimulated microglia induce neuron death via synergistic stimulation of tumor necrosis factor alpha and NMDA receptors. J Neurosci 25:2566-2575.

Gao HM, Hong JS (2008) Why neurodegenerative diseases are progressive: uncontrolled inflammation drives disease progression. Trends Immunol 29:357-365.

Gao HM, Hong JS, Zhang W, Liu B (2002a) Distinct role for microglia in rotenone-induced degeneration of dopaminergic neurons. J Neurosci 22:782-790.

Gao HM, Jiang J, Wilson B, Zhang W, Hong JS, Liu B (2002b) Microglial activation-mediated delayed and progressive degeneration of rat nigral dopaminergic neurons: relevance to Parkinson's disease. J Neurochem 81:1285-1297.

Gao HM, Liu B, Zhang W, Hong JS (2003a) Critical role of microglial NADPH oxidase-derived free radicals in the in vitro MPTP model of Parkinson's disease. FASEB J 17:1954-1956.

Gao HM, Liu B, Zhang W, Hong JS (2003b) Novel anti-inflammatory therapy for Parkinson's disease. Trends Pharmacol Sci 24:395-401.

Gayle DA, Ling Z, Tong C, Landers T, Lipton JW, Carvey PM (2002) Lipopolysaccharide (LPS)-induced dopamine cell loss in culture: roles of tumor necrosis factor-alpha, interleukin-1beta, and nitric oxide. Brain Res Dev Brain Res 133:27-35.

Glass CK, Saijo K, Winner B, Marchetto MC, Gage FH (2010) Mechanisms underlying inflammation in neurodegeneration. Cell 140:918-934.

Goodwin JL, Kehrli ME Jr, Uemura E (1997) Integrin Mac-1 and betaamyloid in microglial release of nitric oxide. Brain Res 768:279-286.

Hirsch EC, Hunot S (2009) Neuroinflammation in Parkinson's disease: a target for neuroprotection? Lancet Neurol 8:382-397.

Hoffmann A, Levchenko A, Scott ML, Baltimore D (2002) The IkappaBNF-kappaB signaling module: temporal control and selective gene activation. Science 298:1241-1245.

Hong Z, Shi M, Chung KA, Quinn JF, Peskind ER, Galasko D, Jankovic J, Zabetian CP, Leverenz JB, Baird G, Montine TJ, Hancock AM, Hwang H, Pan C, Bradner J, Kang UJ, Jensen PH, Zhang J (2010) DJ-1 and alphasynuclein in human cerebrospinal fluid as biomarkers of Parkinson's disease. Brain 133:713-726.

Hu X, Zhang D, Pang H, Caudle WM, Li Y, Gao H, Liu Y, Qian L, Wilson B, Di Monte DA, Ali SF, Zhang J, Block ML, Hong JS (2008) Macrophage antigen complex-1 mediates reactive microgliosis and progressive dopaminergic neurodegeneration in the MPTP model of Parkinson's disease. J Immunol 181:7194-7204.

Kim JB, Sig Choi J, Yu YM, Nam K, Piao CS, Kim SW, Lee MH, Han PL, Park JS, Lee JK (2006) HMGB1, a novel cytokine-like mediator linking acute neuronal death and delayed neuroinflammation in the postischemic brain. J Neurosci 26:6413-6421.

Kordower JH, Chu Y, Hauser RA, Freeman TB, Olanow CW (2008) Lewy body-like pathology in long-term embryonic nigral transplants in Parkinson's disease. Nat Med 14:504-506.

Kreutzberg GW (1996) Microglia: a sensor for pathological events in the CNS. Trends Neurosci 19:312-318.

Langston JW, Forno LS, Tetrud J, Reeves AG, Kaplan JA, Karluk D (1999) Evidence of active nerve cell degeneration in the substantia nigra of humans years after 1-methyl-4-phenyl-1,2,3,6-tetrahydropyridine exposure. Ann Neurol 46:598-605.

Le Cabec V, Carréno S, Moisand A, Bordier C, Maridonneau-Parini I (2002) Complement receptor 3 (CD11b/CD18) mediates type I and type II phagocytosis during nonopsonic and opsonic phagocytosis, respectively. J Immunol 169:2003-2009. 
Li JY, Englund E, Holton JL, Soulet D, Hagell P, Lees AJ, Lashley T, Quinn NP, Rehncrona S, Björklund A, Widner H, Revesz T, Lindvall O, Brundin P (2008) Lewy bodies in grafted neurons in subjects with Parkinson's disease suggest host-to-graft disease propagation. Nat Med 14:501-503.

Liberatore GT, Jackson-Lewis V, Vukosavic S, Mandir AS, Vila M, McAuliffe WG, Dawson VL, Dawson TM, Przedborski S (1999) Inducible nitric oxide synthase stimulates dopaminergic neurodegeneration in the MPTP model of Parkinson disease. Nat Med 5:1403-1409.

Ling Z, Zhu Y, Tong C, Snyder JA, Lipton JW, Carvey PM (2006) Progressive dopamine neuron loss following supra-nigral lipopolysaccharide (LPS) infusion into rats exposed to LPS prenatally. Exp Neurol 199: 499-512.

Liu B, Gao HM, Hong JS (2003) Parkinson's disease and exposure to infectious agents and pesticides and the occurrence of brain injuries: role of neuroinflammation. Environ Health Perspect 111:1065-1073.

Lotze MT, Tracey KJ (2005) High-mobility group box 1 protein (HMGB1): nuclear weapon in the immune arsenal. Nat Rev Immunol 5:331-342.

Lynch OT, Giembycz MA, Barnes PJ, Hellewell PG, Lindsay MA (1999) "Outside-in" signalling mechanisms underlying CD11b/CD18-mediated NADPH oxidase activation in human adherent blood eosinophils. Br J Pharmacol 128:1149-1158.

Maroso M, Balosso S, Ravizza T, Liu J, Aronica E, Iyer AM, Rossetti C, Molteni M, Casalgrandi M, Manfredi AA, Bianchi ME, Vezzani A (2010) Toll-like receptor 4 and high-mobility group box- 1 are involved in ictogenesis and can be targeted to reduce seizures. Nat Med 16:413-419.

Martinez-Vicente M, Talloczy Z, Kaushik S, Massey AC, Mazzulli J, Mosharov EV, Hodara R, Fredenburg R, Wu DC, Follenzi A, Dauer W, Przedborski S, Ischiropoulos H, Lansbury PT, Sulzer D, Cuervo AM (2008) Dopamine-modified alpha-synuclein blocks chaperone-mediated autophagy. J Clin Invest 118:777-788.

Mayadas TN, Cullere X (2005) Neutrophil beta2 integrins: moderators of life or death decisions. Trends Immunol 26:388-395.

McGeer EG, McGeer PL (2003) Inflammatory processes in Alzheimer's disease. Prog Neuropsychopharmacol Biol Psychiatry 27:741-749.

McGeer PL, Itagaki S, Boyes BE, McGeer EG (1988) Reactive microglia are positive for HLA-DR in the substantia nigra of Parkinson's and Alzheimer's disease brains. Neurology 38:1285-1291.

McGeer PL, Schwab C, Parent A, Doudet D (2003) Presence of reactive microglia in monkey substantia nigra after 1-methyl-4-phenyl-1,2,3,6tetrahydropyridine administration. Ann Neurol 54:599-604.

McNaught KS, Olanow CW (2006) Protein aggregation in the pathogenesis of familial and sporadic Parkinson's disease. Neurobiol Aging 27:530-545.

Mendez I, Viñuela A, Astradsson A, Mukhida K, Hallett P, Robertson H, Tierney T, Holness R, Dagher A, Trojanowski JQ, Isacson O (2008) Dopamine neurons implanted into people with Parkinson's disease survive without pathology for 14 years. Nat Med 14:507-509.

Mittal D, Saccheri F, Vénéreau E, Pusterla T, Bianchi ME, Rescigno M (2010) TLR4-mediated skin carcinogenesis is dependent on immune and radioresistant cells. EMBO J 29:2242-2252.

Mogi M, Harada M, Kondo T, Riederer P, Inagaki H, Minami M, Nagatsu T (1994) Interleukin-1 beta, interleukin-6, epidermal growth factor and transforming growth factor-alpha are elevated in the brain from parkinsonian patients. Neurosci Lett 180:147-150.

Monahan AJ, Warren M, Carvey PM (2008) Neuroinflammation and peripheral immune infiltration in Parkinson's disease: an autoimmune hypothesis. Cell Transplant 17:363-372.

Muhammad S, Barakat W, Stoyanov S, Murikinati S, Yang H, Tracey KJ, Bendszus M, Rossetti G, Nawroth PP, Bierhaus A, Schwaninger M (2008) The HMGB1 receptor RAGE mediates ischemic brain damage. J Neurosci 28:12023-12031.

Nguyen MD, Julien JP, Rivest S (2002) Innate immunity: the missing link in neuroprotection and neurodegeneration? Nat Rev Neurosci 3:216-227.
Pei Z, Pang H, Qian L, Yang S, Wang T, Zhang W, Wu X, Dallas S, Wilson B, Reece JM, Miller DS, Hong JS, Block ML (2007) MAC1 mediates LPSinduced production of superoxide by microglia: the role of pattern recognition receptors in dopaminergic neurotoxicity. Glia 55:1362-1373.

Przedborski S (2010) Inflammation and Parkinson's disease pathogenesis. Mov Disord 25 [Suppl 1]:S55-S57.

Qin L, Wu X, Block ML, Liu Y, Breese GR, Hong JS, Knapp DJ, Crews FT (2007) Systemic LPS causes chronic neuroinflammation and progressive neurodegeneration. Glia 55:453-462.

Ross GD (2000) Regulation of the adhesion versus cytotoxic functions of the Mac-1/CR3/alphaMbeta2-integrin glycoprotein. Crit Rev Immunol 20:197-222.

Ross GD, Větvicka V (1993) CR3 (CD11b, CD18): a phagocyte and NK cell membrane receptor with multiple ligand specificities and functions. Clin Exp Immunol 92:181-184.

Scaffidi P, Misteli T, Bianchi ME (2002) Release of chromatin protein HMGB1 by necrotic cells triggers inflammation. Nature 418:191-195.

Schwartz M (2002) Autoimmunity as the body's defense mechanism against the enemy within: development of therapeutic vaccines for neurodegenerative disorders. J Neurovirol 8:480-485.

Seong SY, Matzinger P (2004) Hydrophobicity: an ancient damageassociated molecular pattern that initiates innate immune responses. Nat Rev Immunol 4:469-478.

Simard AR, Soulet D, Gowing G, Julien JP, Rivest S (2006) Bone marrowderived microglia play a critical role in restricting senile plaque formation in Alzheimer's disease. Neuron 49:489-502.

Sims GP, Rowe DC, Rietdijk ST, Herbst R, Coyle AJ (2010) HMGB1 and RAGE in inflammation and cancer. Annu Rev Immunol 28:367-388.

Takata K, Kitamura Y, Tsuchiya D, Kawasaki T, Taniguchi T, Shimohama S (2004) High mobility group box protein-1 inhibits microglial Abeta clearance and enhances Abeta neurotoxicity. J Neurosci Res 78:880-891.

Tian L, Rauvala H, Gahmberg CG (2009) Neuronal regulation of immune responses in the central nervous system. Trends Immunol 30:91-99.

Wahner AD, Sinsheimer JS, Bronstein JM, Ritz B (2007) Inflammatory cytokine gene polymorphisms and increased risk of Parkinson disease. Arch Neurol 64:836-840.

Wang L, Duke L, Zhang PS, Arlinghaus RB, Symmans WF, Sahin A, Mendez R, Dai JL (2003) Alternative splicing disrupts a nuclear localization signal in spleen tyrosine kinase that is required for invasion suppression in breast cancer. Cancer Res 63:4724-4730.

Wright SD, Jong MT (1986) Adhesion-promoting receptors on human macrophages recognize Escherichia coli by binding to lipopolysaccharide. J Exp Med 164:1876-1888.

Wyss-Coray T, Mucke L (2002) Inflammation in neurodegenerative disease-a double-edged sword. Neuron 35:419-432.

Yamada T, McGeer PL, McGeer EG (1992) Lewy bodies in Parkinson's disease are recognized by antibodies to complement proteins. Acta Neuropathol 84:100-104.

Zhang F, Qian L, Flood PM, Shi JS, Hong JS, Gao HM (2010) Inhibition of IкB kinase- $\beta($ IKK- $\beta$ ) protects dopamine neurons against lipopolysaccharideinduced neurotoxicity. J Pharmacol Exp Ther 333:822-833.

Zhang G, Ghosh S (2001) Toll-like receptor-mediated NF-kappaB activation: a phylogenetically conserved paradigm in innate immunity. J Clin Invest 107:13-19.

Zhang W, Dallas S, Zhang D, Guo JP, Pang H, Wilson B, Miller DS, Chen B, Zhang W, McGeer PL, Hong JS, Zhang J (2007) Microglial PHOX and Mac-1 are essential to the enhanced dopaminergic neurodegeneration elicited by A30P and A53T mutant alpha-synuclein. Glia 55:1178-1188.

Zhou C, Huang Y, Przedborski S (2008) Oxidative stress in Parkinson's disease: a mechanism of pathogenic and therapeutic significance. Ann N Y Acad Sci 1147:93-104. 